DO-TH 2002/08

hep-ph/0205301

May 2002

\title{
Two-Photon Processes and Photon Structure
}

\author{
I. Schienbein \\ Universität Dortmund, Institut für Physik, \\ D-44221 Dortmund, Germany
}

\begin{abstract}
In this article aspects of photon-photon physics related to the structure of real and virtual photons are reviewed. A re-calculation of the virtual photon-photon box is performed and some discrepancies in the literature are clarified. A useful compilation of various relevant limits derived from the most general expressions is provided. Furthermore, structure functions of spin-averaged, transverse and longitudinal virtual target photons are defined and discussed. Finally, the factorization of two-photon processes in the Bjorken limit is demonstrated to hold also for the case of virtual target photons.
\end{abstract}

Key Words: two-photon processes, photon structure, photon-photon box, doubly virtual box, quark parton model, virtual photons, photon structure functions, factorization 


\section{INTRODUCTION}

At present, two-photon processes $e^{+} e^{-} \rightarrow e^{+} e^{-} \gamma^{\star} \gamma^{\star} \rightarrow e^{+} e^{-} X$ in $e^{+} e^{-}$ collider experiments are the main source of information on the (hadronic and QED) structure of the photon. As has been first realized in [1] such processes factorize in the Bjorken limit into a flux of (quasi-)real target photons which can then be probed in deep inelastic electron-photon scattering $\left(\mathrm{DIS}_{e \gamma}\right)$. Analogously to the familiar deep inelastic $e p$ scattering, the unpolarized cross section for $\mathrm{DIS}_{e \gamma}, e \gamma \rightarrow e X$, can be described in terms of two independent photon structure functions, say $F_{2}^{\gamma}\left(x, Q^{2}\right)$ and $F_{L}^{\gamma}\left(x, Q^{2}\right)$. (There are two more structure functions in the case of a (quasi-)real target photon: $g_{1}^{\gamma}\left(x, Q^{2}\right)$ which can be measured in polarized DIS $_{e \gamma}$ again completely analogous to the case of deep inelastic scattering off spin- $1 / 2$ targets like protons and a further structure function involving helicity-flips of the target photon's helicity which is accessible by the azimuthal angular dependence of the $e^{+} e^{-}$cross section.) Due to experimental constraints the DIS $_{e \gamma}$ cross sections are measured at small values of the variable $y$ (defined as usual) where $F_{L}^{\gamma}\left(x, Q^{2}\right)$ is kinematically suppressed due to a pre-factor $\propto y^{2}$ in the cross section and it is the structure function $F_{2}^{\gamma}\left(x, Q^{2}\right)$ which is measured. The DIS $\operatorname{Dit}_{\text {e }}$ data on the photon structure function $F_{2}^{\gamma}\left(x, Q^{2}\right)$ are mainly sensitive to the up-quark density $u^{\gamma}\left(x, Q^{2}\right)$ in the photon as can be seen from the parton model expression for $F_{2}^{\gamma}$ (in LO), $F_{2}^{\gamma} \propto 4 u^{\gamma}+d^{\gamma}+s^{\gamma}$, whereas the gluon distribution $g^{\gamma}\left(x, Q^{2}\right)$ is only indirectly constrained at small values of $x$ due to the evolution. Complementary information has become available in the last years due to 'resolved photon processes' (e.g. production of (di-)jets or hadrons with large $p_{T}\left(E_{T}\right)$, heavy quark production, isolated prompt photon production) in $\gamma p$ and $\gamma \gamma$ collisions at the $e p$ collider HERA and the $e^{+} e^{-}$collider LEP, respectively, which are mainly sensitive to the gluon distribution in the photon. Triggered by the wealth of experimental results from the $e^{+} e^{-}$collider LEP and the $e p$ collider HERA $[2,3]$ which became available in the last decade several QCD analyses of photonic parton distributions $[4,5,6,7,8]$ have been performed. Recently some effort has also been devoted to the polarized structure function $g_{1}^{\gamma}$ $[9,10,11]$. The polarized parton distributions $\Delta f^{\gamma}\left(x, Q^{2}\right)$ will be measurable at future polarized $e^{+} e^{-}$and $e p$ colliders, see [12] and references therein.

So far, the discussion has referred to the most important case of (quasi)real photon targets. However, there are good reasons for considering the general case of virtual photon targets $\gamma\left(P^{2}\right)$ where $P^{2}$ is the virtuality of the target photon: First of all, at present $e^{+} e^{-}$and $e p$ collider experiments the photon beams consist of bremsstrahlung radiated off the incident lepton beam resulting in a continuous spectrum of target photons $\gamma\left(P^{2}\right)$. The bremsstrahlung spectrum is proportional to $1 / P^{2}$ such that the bulk of target photons is produced at $P^{2} \simeq P_{\min }^{2} \simeq 0$. The parton content of such (quasi-)real photons is well established both experimen- 
tally and theoretically and in general one expects $[13,14,15,6,8]$ also virtual photons to possess a parton content smoothly depending on $P^{2}$. In this sense the real photon $\gamma \equiv \gamma\left(P^{2} \simeq 0\right)$ is just a 'primus inter pares' and unified approaches to the parton content of virtual photons $\gamma\left(P^{2}\right)$ which comprise the real photon case in the limit $P^{2} \rightarrow 0[8,15,6,11]$ are highly desirable. This is also reflected by the fact that measurements of the real photon structure function $F_{2}^{\gamma}$ in single-tag events integrate over the bremsstrahlung spectrum from $P_{\min }^{2}$ up to a $P_{\max }^{2}$ which depends on the experimental details. For instance, at LEP1(LEP2) $P_{\max }^{2}$ is as large as $P_{\max }^{2} \simeq 1.5 \mathrm{GeV}^{2}\left(4.5 \mathrm{GeV}^{2}\right)$, cf. Section 2.2 in [2]. Although the bulk of photons is produced at $P^{2} \simeq P_{\min }^{2}$ the amount of ignorance of the $P^{2}$ dependence, mainly in the range $P^{2} \lesssim \Lambda^{2}$, where $\Lambda$ is a typical hadronic scale, feeds back on the determination of the structure function $F_{2}^{\gamma}$ (parton distributions) of (quasi-)real photons. Furthermore, the case of deeply virtual target photons $Q^{2} \gg P^{2} \gg \Lambda^{2}$ has attracted a lot of theoretical interest in the unpolarized $[16,17,18,19]$ as well as in the polarized case $[20,11]$ because it is purely perturbative allowing for absolute QCD predictions.

The aim of this work is to review aspects of two-photon physics related to the structure of real and virtual photons. The description of virtual target photons necessitates a more involved theoretical framework due to the longitudinal photon polarization and the more complicated kinematics. The paper is organized as follows: In Section 2 we give a comprehensible introduction into two-photon processes. After defining the required kinematical variables, we discuss the 4-photon amplitude and derive the general cross section for two-photon processes. Furthermore, we perform a re-calculation of the doubly virtual box $\gamma^{\star}\left(Q^{2}\right) \gamma^{\star}\left(P^{2}\right) \rightarrow q \bar{q}$ in lowest order perturbation theory. Thereby we clarify two discrepancies in the literature $[21,18]$. The general results of this calculation are casted in a form which easily allows to read off various important limits, e.g., the quark-parton model (QPM) results for the structure functions of real and virtual photons and the heavy quark contributions to the photon structure functions. A useful compilation of these limits can be found in Appendix A. The notation of this section follows mainly the report of Budnev et al. [21]. Next, in Sec. 3 we define structure functions for virtual photons. Beside the spin-averaged target usually considered in the literature we discuss the possibility of defining structure functions for transverse and longitudinal target photons in view of the recent approaches in the literature to the parton content of virtual longitudinal photons [22,23]. This will also be of importance in Sec. 4 where we investigate the factorization of two-photon processes in the 'generalized' Bjorken limit $P^{2} \neq 0, Q^{2} \rightarrow \infty, x=$ fixed. As we will see, the cross section factorizes also in this case of non-zero virtualities $P^{2}$ of the 'target photon' into fluxes of transversely and longitudinally polarized photons times the corresponding cross sections for deep inelastic scattering off these target photons up to terms of the order $\mathcal{O}\left(\sqrt{P^{2} / Q^{2}}\right)$. 
It should be noted that the factorization is essential for a theoretical description of two-photon processes in terms of (virtual) photon structure functions which can be measured in deep inelastic electron-(virtual) photon scattering. Finally, in Sec. 5 we summarize the main results and draw some conclusions.

\section{TWO-PHOTON PROCESSES}

\subsection{Kinematics}

The kinematics of particle production via photon-photon scattering in $e^{+} e^{-}$collisions

$$
e^{-}\left(p_{1}\right) e^{+}\left(p_{2}\right) \rightarrow e^{-}\left(p_{1}^{\prime}\right) e^{+}\left(p_{2}^{\prime}\right) \gamma^{\star}(q) \gamma^{\star}(p) \rightarrow e^{-}\left(p_{1}^{\prime}\right) e^{+}\left(p_{2}^{\prime}\right) \mathrm{X}\left(p_{\mathrm{X}}\right)
$$

is depicted in Fig. 1. The momenta of the incoming and outgoing leptons

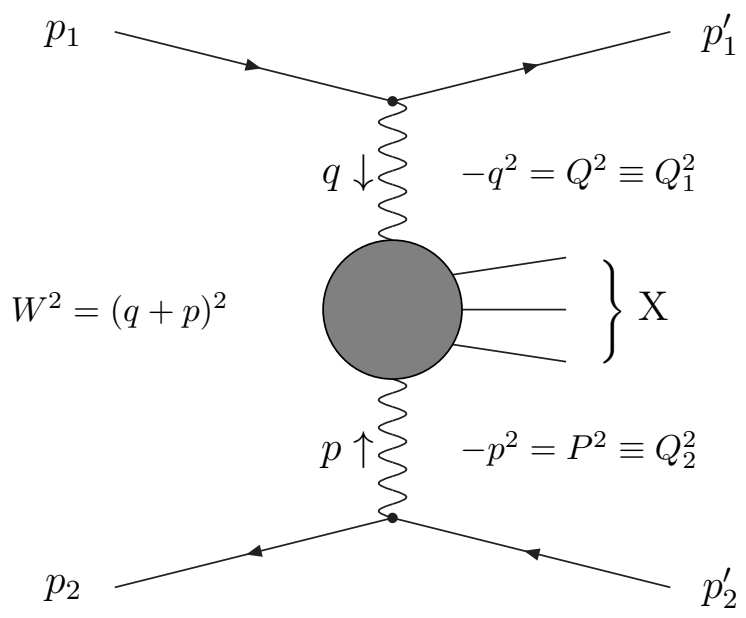

FIG. 1 Two-photon particle production. The solid lines are the incoming and outgoing leptons and the wavy lines are virtual photons which produce a final state X consisting of hadrons (or leptons).

are denoted by $p_{i} \equiv\left(E_{i}, \overrightarrow{p_{i}}\right)$ and $p_{i}^{\prime} \equiv\left(E_{i}^{\prime}, \vec{p}_{i}^{\prime}\right)(i=1,2)$ respectively and the momenta of the photons are given by

$$
\begin{array}{ll}
q \equiv p_{1}-p_{1}^{\prime}, & Q^{2}=-q^{2} \equiv Q_{1}^{2}, \\
p \equiv p_{2}-p_{2}^{\prime}, & P^{2}=-p^{2} \equiv Q_{2}^{2} .
\end{array}
$$


In general both photons have space-like momenta and $P^{2}$ refers to the photon with smaller virtuality $\left(P^{2} \leq Q^{2}\right)$. X denotes the final state produced in the $\gamma^{\star}(q)+\gamma^{\star}(p) \rightarrow \mathrm{X}$ subprocess. For later use, we define the following variables:

$$
\begin{gathered}
\nu=p \cdot q, \quad x=\frac{Q^{2}}{2 \nu}, \quad \delta=\frac{P^{2}}{2 \nu}, \quad y_{1}=\frac{p \cdot q}{p \cdot p_{1}}, \quad y_{2}=\frac{p \cdot q}{q \cdot p_{2}}, \\
W^{2} \equiv(p+q)^{2}=2 \nu(1-x-\delta)=Q^{2} \frac{1-x}{x}-P^{2}
\end{gathered}
$$

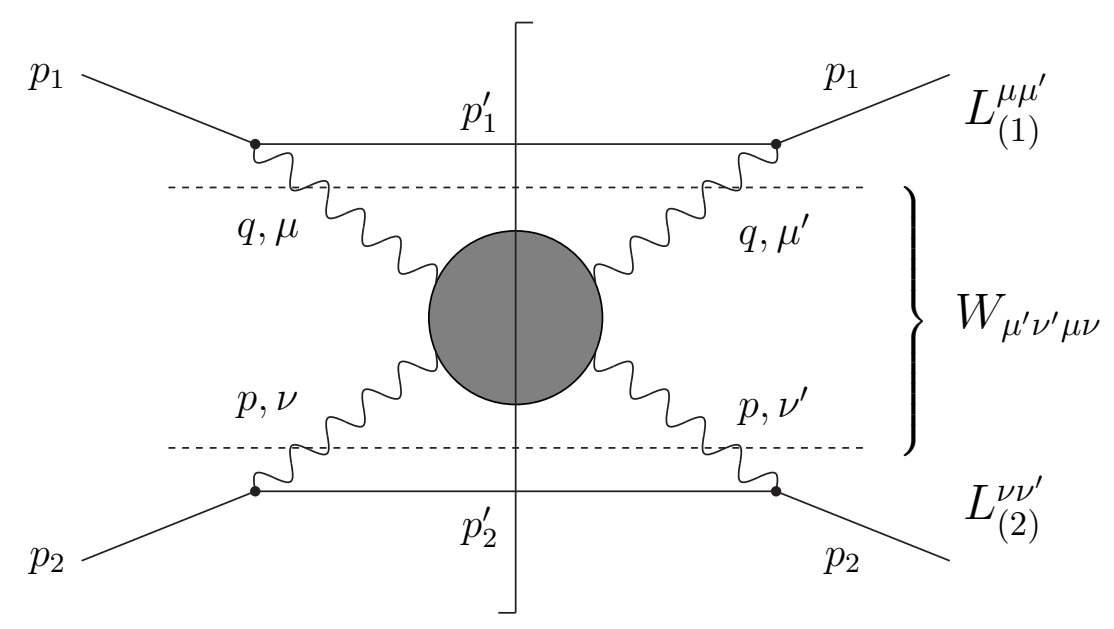

FIG. 2 Squared matrix element of the process (2.1). Integration over the phase space of the system $\mathrm{X}$ is implied as indicated by the vertical cut.

It should be noted that two-photon processes comprise only a subset of Feynman diagrams contributing to the physically observable process $e^{+} e^{-} \rightarrow e^{+} e^{-} X$. Other contributions arise from $t$ - and $s$-channel bremsstrahlung diagrams where the bremsstrahlung photon produces the hadronic final state (see, e.g., Fig. 1 and the discussion in [24]) and from diagrams where one (or both) of the photons is replaced by a $Z$ boson. However, applying appropriate cuts these contributions are negligible. At first, the $s$-channel diagram is suppressed by a factor $1 / s$ and is therefore very small. Furthermore, $Z$-exchange can be neglected as long as the boson virtualities are not too large, say $Q^{2}<500 \mathrm{GeV}^{2}$. On the other hand, the $t$-channel bremsstrahlung contribution deserves more care in certain kinematical regions. However, as long as the scattering angle of at least one of the leptons is small the direct two-photon process is strongly enhanced by a collinear logarithm $\ln E / m_{e}$ such that the cross section for 
$e^{+} e^{-} \rightarrow e^{+} e^{-} X$ is dominated by the two-photon mechanism. In the following we do not distinguish between two-photon processes and the measurable $e^{+} e^{-} \rightarrow e^{+} e^{-} X$.

The cross section for the process (2.1) is given by

$$
d \sigma=\frac{1}{F_{e e}}|M|^{2} d Q^{(n+2)}
$$

with the invariant matrix element $M$, the Møller flux factor of the two incoming leptons

$$
F_{e e}=4 \sqrt{\left(p_{1} \cdot p_{2}\right)^{2}-m_{e}^{2} m_{e}^{2}},
$$

and the Lorentz-invariant $(n+2)$-particle phase space

$$
\begin{aligned}
d Q^{(n+2)}( & \left.p_{1}+p_{2} ; p_{1}^{\prime}, p_{2}^{\prime}, k_{1}, \ldots, k_{n}\right) \\
& =\frac{d^{3} p_{1}^{\prime}}{(2 \pi)^{3} 2 E_{1}^{\prime}} \frac{d^{3} p_{2}^{\prime}}{(2 \pi)^{3} 2 E_{2}^{\prime}} d Q^{(n)}\left(q+p ; k_{1}, \ldots, k_{n}\right) \\
& =\frac{d^{3} p_{1}^{\prime}}{(2 \pi)^{3} 2 E_{1}^{\prime}} \frac{d^{3} p_{2}^{\prime}}{(2 \pi)^{3} 2 E_{2}^{\prime}}(2 \pi)^{4} \delta^{(4)}\left(q+p-p_{\mathrm{X}}\right) d \Gamma .
\end{aligned}
$$

Here $p_{\mathrm{X}}=\sum_{i} k_{i}, i \in \mathrm{X}$ is the total momentum and $d \Gamma=\prod_{i} \frac{d^{3} k_{i}}{2 k_{i}^{0}(2 \pi)^{3}}, i \in \mathrm{X}$ the phase space volume of the produced system X.

The cross section can be expressed in terms of the amplitudes $M^{\mu \nu}$ of the $\gamma^{\star}(q)+\gamma^{\star}(p) \rightarrow \mathrm{X}$ subprocess as follows (see Fig. 2):

$$
d \sigma=\frac{d^{3} p_{1}^{\prime} d^{3} p_{2}^{\prime}}{2 E_{1}^{\prime} 2 E_{2}^{\prime}(2 \pi)^{6}} \frac{(4 \pi \alpha)^{2}}{Q^{4} P^{4}} \frac{1}{F_{e e}} L_{(1)}^{\mu \mu^{\prime}} L_{(2)}^{\nu \nu^{\prime}} W_{\mu^{\prime} \nu^{\prime}, \mu \nu}
$$

with

$$
W^{\mu^{\prime} \nu^{\prime}, \mu \nu}=\frac{1}{2} \int M^{\star \mu^{\prime} \nu^{\prime}} M^{\mu \nu}(2 \pi)^{4} \delta^{(4)}\left(q+p-p_{\mathrm{X}}\right) d \Gamma .
$$

For unpolarized leptons the (leptonic) tensors $L_{(1)}$ and $L_{(2)}$ (see Fig. 2) are given by

$$
L_{(i)}^{\alpha \beta}=\frac{1}{2} \operatorname{Tr}\left[\left(\not p_{i}+m_{\mathrm{e}}\right) \gamma^{\alpha}\left(\not \not_{i}^{\prime}+m_{\mathrm{e}}\right) \gamma^{\beta}\right] .
$$

The factor $1 / 2$ is due to a spin average over the incoming leptons. In addition, we introduce the dimensionless quantities [21]

$$
\rho_{\mathrm{i}}^{\alpha \beta}=\frac{1}{Q_{i}^{2}} L_{(i)}^{\alpha \beta}=-\left(g^{\alpha \beta}-\frac{q_{i}^{\alpha} q_{i}^{\beta}}{q_{i}^{2}}\right)-\frac{\left(2 p_{i}-q_{i}\right)^{\alpha}\left(2 p_{i}-q_{i}\right)^{\beta}}{q_{i}^{2}}
$$

which have the interpretation of (unnormalized) density matrices for the corresponding virtual photons. 


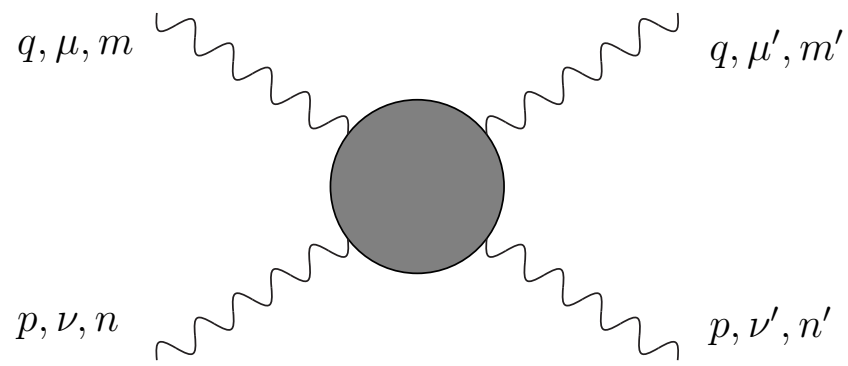

FIG. 3 The photon-photon forward scattering amplitude $T^{\mu^{\prime} \nu^{\prime}, \mu \nu} ; m, n$, $m^{\prime}$, and $n^{\prime}$ are helicity indices. The tensor $W^{\mu^{\prime} \nu^{\prime}, \mu \nu}$ defined in (2.8) is the absorptive part of the $\gamma \gamma$ forward amplitude: $W^{\mu^{\prime} \nu^{\prime}, \mu \nu}=\frac{1}{\pi} \operatorname{Im} T^{\mu^{\prime} \nu^{\prime}, \mu \nu}$.

\subsection{The Hadronic Tensor $W^{\mu^{\prime} \nu^{\prime}, \mu \nu}$}

According to the optical theorem $W^{\mu^{\prime} \nu^{\prime}, \mu \nu}$ is the absorptive part of the virtual $\gamma \gamma$ forward amplitude shown in Fig. 3. Taking into account P- and T-invariance (symmetry $\mu^{\prime} \nu^{\prime} \leftrightarrow \mu \nu$ ) and gauge invariance, i.e.

$$
q_{\mu} W^{\mu^{\prime} \nu^{\prime}, \mu \nu}=q_{\mu^{\prime}} W^{\mu^{\prime} \nu^{\prime}, \mu \nu}=p_{\nu} W^{\mu^{\prime} \nu^{\prime}, \mu \nu}=p_{\nu^{\prime}} W^{\mu^{\prime} \nu^{\prime}, \mu \nu}=0,
$$

the Lorentz-tensor $W^{\mu^{\prime} \nu^{\prime}, \mu \nu}$ can be expanded in terms of a basis of 8 independent tensors constructed from the vectors $q, p$ and the metric tensor $g$. The choice of the tensor basis in which the expansion is carried out is clearly arbitrary and different forms are discussed in the literature [21, 25, 26]. In the following we will stick to the very physical expansion given in [21]:

$$
\begin{aligned}
W^{\mu^{\prime} \nu^{\prime}, \mu \nu} & =R^{\mu^{\prime} \mu} R^{\nu^{\prime} \nu} W_{\mathrm{TT}}+R^{\mu^{\prime} \mu} Q_{2}^{\nu} Q_{2}^{\nu^{\prime}} W_{\mathrm{TL}}+Q_{1}^{\mu^{\prime}} Q_{1}^{\mu} R^{\nu^{\prime} \nu} W_{\mathrm{LT}} \\
& +Q_{1}^{\mu^{\prime}} Q_{1}^{\mu} Q_{2}^{\nu^{\prime}} Q_{2}^{\nu} W_{\mathrm{LL}} \\
& +\frac{1}{2}\left(R^{\mu^{\prime} \nu^{\prime}} R^{\mu \nu}+R^{\mu^{\prime} \nu} R^{\nu^{\prime} \mu}-R^{\mu^{\prime} \mu} R^{\nu^{\prime} \nu}\right) W_{\mathrm{TT}}^{\tau} \\
& -\left(R^{\mu \nu} Q_{1}^{\mu^{\prime}} Q_{2}^{\nu^{\prime}}+R^{\nu^{\prime} \mu} Q_{1}^{\mu^{\prime}} Q_{2}^{\nu}+R^{\mu^{\prime} \nu^{\prime}} Q_{1}^{\mu} Q_{2}^{\nu}+R^{\mu^{\prime} \nu} Q_{2}^{\nu^{\prime}} Q_{1}^{\mu}\right) W_{\mathrm{TL}}^{\tau} \\
& +\left(R^{\mu^{\prime} \nu^{\prime}} R^{\mu \nu}-R^{\nu^{\prime} \mu} R^{\mu^{\prime} \nu}\right) W_{\mathrm{TT}}^{\mathrm{a}} \\
& -\left(R^{\mu \nu} Q_{1}^{\mu^{\prime}} Q_{2}^{\nu^{\prime}}-R^{\nu^{\prime} \mu} Q_{1}^{\mu^{\prime}} Q_{2}^{\nu}+R^{\mu^{\prime} \nu^{\prime}} Q_{1}^{\mu} Q_{2}^{\nu}-R^{\mu^{\prime} \nu} Q_{2}^{\nu^{\prime}} Q_{1}^{\mu}\right) W_{\mathrm{TL}}^{\mathrm{a}}
\end{aligned}
$$

with $\bar{\beta}^{2} \equiv 1-4 x \delta$ and

$$
\begin{gathered}
R^{\alpha \beta}=-g^{\alpha \beta}+\frac{\nu\left(p^{\alpha} q^{\beta}+q^{\alpha} p^{\beta}\right)-p^{2} q^{\alpha} q^{\beta}-q^{2} p^{\alpha} p^{\beta}}{\nu^{2} \bar{\beta}^{2}} \\
Q_{1}^{\alpha}=\frac{\sqrt{-q^{2}}}{\nu \bar{\beta}}\left(p^{\alpha}-\frac{\nu}{q^{2}} q^{\alpha}\right), Q_{2}^{\alpha}=\frac{\sqrt{-p^{2}}}{\nu \bar{\beta}}\left(q^{\alpha}-\frac{\nu}{p^{2}} p^{\alpha}\right) .
\end{gathered}
$$




\subsubsection{Construction of the Tensor}

Since it is quite insightful we now describe the main ideas of the construction of the photon tensor from the photon photon helicity amplitudes [21] which have simple physical interpretations. A nice discussion of the helicity amplitude formalism can also be found in Ref. [27].

In Fig. $3 m, n$ and $m^{\prime}, n^{\prime}$ are the helicities of the initial state and final state photons, respectively, which can adopt the values $m, n, m^{\prime}, n^{\prime}=0, \pm 1$ and are constrained by angular momentum conservation: $m^{\prime}-n^{\prime}=m-n$. The helicity amplitudes $W_{m^{\prime} n^{\prime}, m n}$ are related to the photon tensor in the following way

$$
W_{m^{\prime} n^{\prime}, m n} \equiv \epsilon_{m^{\prime}}^{\mu^{\prime}}(q)^{\star} \epsilon_{n^{\prime}}^{\nu^{\prime}}(p)^{\star} W_{\mu^{\prime} \nu^{\prime}, \mu \nu} \epsilon_{m}^{\mu}(q) \epsilon_{n}^{\nu}(p) .
$$

Due to $\mathrm{P}$ - and T-invariance and helicity conservation we have

$W_{m^{\prime} n^{\prime}, m n} \stackrel{P}{=}(-1)^{m^{\prime}-n^{\prime}+m-n} W_{-m^{\prime}-n^{\prime},-m-n}=W_{-m^{\prime}-n^{\prime},-m-n} \stackrel{T}{=} W_{m n, m^{\prime} n^{\prime}}$

such that there are 8 independent helicity amplitudes, say $W_{++,++}, W_{+-,+-}$, $W_{+0,+0}, W_{0+, 0+}, W_{00,00}, W_{++,--}, W_{++, 00}, W_{0+,-0}$ where the latter three involve helicity flips. In addition, there are three independent positivity constraints on these amplitudes due to the Cauchy-Schwarz inequality $\left|W_{m^{\prime} n^{\prime}, m n}\right| \leq \sqrt{W_{m^{\prime} n^{\prime}, m^{\prime} n^{\prime}} W_{m n, m n}}[28]$. For instance, we find for the above helicity amplitudes the relations $\left|W_{++,--}\right| \leq W_{++,++},\left|W_{++, 00}\right| \leq$ $\sqrt{W_{++,++} W_{00,00}}$, and $\left|W_{0+,-0}\right| \leq \sqrt{W_{+0,+0} W_{0+, 0+}}$. Due to the completeness and orthonormality relations for (space-like) polarization vectors Eq. (2.13) can be 'inverted' resulting in a very nice representation of $W_{\mu^{\prime} \nu^{\prime}, \mu \nu}$ through the helicity amplitudes:

$W^{\mu^{\prime} \nu^{\prime}, \mu \nu}=\sum_{m^{\prime}, n^{\prime}, m, n} C\left(m^{\prime}, n^{\prime}, m, m\right) \epsilon_{m^{\prime}}^{\mu^{\prime}}(q) \epsilon_{n^{\prime}}^{\nu^{\prime}}(p) \epsilon_{m}^{\mu}(q)^{\star} \epsilon_{n}^{\nu}(p)^{\star} W_{m^{\prime} n^{\prime}, m n}$

where for a space-like target $C\left(m^{\prime}, n^{\prime}, m, m\right)=(-1)^{m^{\prime}+n^{\prime}+m+n}=1$. Note that gauge invariance is satisfied by construction due to $q \cdot \epsilon(q)=p \cdot \epsilon(p)=0$. Now the photon polarization vectors in the $\gamma \gamma$-CMS can be written in a covariant way, see App. B and C in [21]:

$$
\begin{gathered}
\epsilon_{0}^{\alpha}(q)=i Q_{1}^{\alpha}, \epsilon_{0}^{\alpha}(p)=-i Q_{2}^{\alpha} \\
\epsilon_{ \pm}^{\star \alpha}(q) \epsilon_{ \pm}^{\beta}(q)=\frac{1}{2}\left[R^{\alpha \beta} \pm i \frac{1}{\nu \bar{\beta}} \varepsilon^{\alpha \beta \rho \sigma} q_{\rho} p_{\sigma}\right], \epsilon_{ \pm}^{\star \alpha}(p) \epsilon_{ \pm}^{\beta}(p)=\epsilon_{\mp}^{\star \alpha}(q) \epsilon_{\mp}^{\beta}(q)
\end{gathered}
$$

with $R^{\alpha \beta}, Q_{1,2}^{\alpha}$ from Eq. (2.12). Using these relations in Eq. (2.14) we arrive at the final tensor given in Eq. (2.11). The dimensionless invariant 
functions $W_{a b}$ depend only on the invariants $W^{2}, Q^{2}$ and $P^{2}$ and are related to the $\gamma \gamma$-helicity amplitudes $W_{m^{\prime} n^{\prime}, m n}$ in the $\gamma \gamma$-CMS via [21]

$$
\begin{array}{rlrl}
W_{\mathrm{TT}} & =\frac{1}{2}\left(W_{++,++}+W_{+-,+-}\right), W_{\mathrm{TL}} & =W_{+0,+0}, \\
W_{\mathrm{LT}} & =W_{0+, 0+}, \\
W_{\mathrm{LL}} & =W_{00,00}, \\
W_{\mathrm{TT}}^{\tau} & =W_{++,--}, \\
W_{\mathrm{TL}}^{\tau} & =\frac{1}{2}\left(W_{++, 00}+W_{0+,-0}\right), \\
W_{\mathrm{TT}}^{\mathrm{a}} & =\frac{1}{2}\left(W_{++,++}-W_{+-,+-}\right), W_{\mathrm{TL}}^{\mathrm{a}}=\frac{1}{2}\left(W_{++, 00}-W_{0+,-0}\right) .
\end{array}
$$

The amplitudes $W_{\mathrm{TT}}^{\tau}, W_{\mathrm{TL}}^{\tau}$, and $W_{\mathrm{TL}}^{\mathrm{a}}$ correspond to transitions with spin flip for each of the photons (with total helicity conservation). As we will see in the next section only 6 of these amplitudes $\left(W_{\mathrm{TT}}, W_{\mathrm{TL}}, W_{\mathrm{LT}}, W_{\mathrm{LL}}, W_{\mathrm{TT}}^{\tau}\right.$, $W_{\mathrm{TL}}^{\tau}$ ) enter the cross section for unpolarized lepton beams because the tensors in (2.10) are symmetric whereas the tensor structures multiplying $W_{\text {TT }}^{\mathrm{a}}$ and $W_{\text {TL }}^{\mathrm{a}}$ in (2.11) are anti-symmetric such that these terms do not contribute when the leptonic and the hadronic tensors are contracted. Only if the initial leptons are polarized, can the amplitudes $W_{\mathrm{TT}}^{\mathrm{a}}$ and $W_{\mathrm{TL}}^{\mathrm{a}}$ be measured as well [21].

Finally, it is noteworthy that the helicity amplitudes obviously satisfy the symmetry [just turn Fig. 3 upside down]

$$
W_{m^{\prime} n^{\prime}, m n}(q, p)=W_{n^{\prime} m^{\prime}, n m}(p, q) .
$$

As a consequence of this and the other above stated symmetries we find that the invariant functions $W_{a b}$ are symmetric under exchanging $q \leftrightarrow p$, i.e. $W_{a b}(q, p)=W_{a b}(p, q)$, with exception of $W_{\mathrm{LT}}$ and $W_{\mathrm{TL}}$ which satisfy $W_{\mathrm{LT}}(q, p)=W_{\mathrm{TL}}(p, q)$.

\subsubsection{Projection Operators}

The photon momenta $q, p$, the unit vectors $Q_{1}, Q_{2}$, and the symmetric tensor $R^{\alpha \beta}$ satisfy the following (orthogonality) relations:

$$
\begin{gathered}
q \cdot Q_{1}=p \cdot Q_{2}=0, Q_{1,2}^{2}=1, \\
q^{\alpha} R_{\alpha \beta}=p^{\alpha} R_{\alpha \beta}=Q_{1,2}^{\alpha} R_{\alpha \beta}=0, R_{\alpha \beta} R^{\alpha \beta}=2, R_{\beta}^{\alpha} R^{\beta \gamma}=-R^{\alpha \gamma} .
\end{gathered}
$$

With help of these relations it is easy to see that the various tensors in front of the invariant functions $W_{a b}$ in Eq. (2.11) are mutually orthogonal and therefore can be used to project out the invariant functions. This is also a direct consequence of the construction in Eq. (2.14) due to the orthonormality of the polarization vectors. With obvious notation $\left(\mathrm{P}_{\mathrm{W}_{\mathrm{TT}} \mu^{\prime} \nu^{\prime}, \mu \nu} W_{\mu^{\prime} \nu^{\prime}, \mu \nu}=W_{\mathrm{TT}}\right.$ etc. $)$ the projectors read:

$$
\mathrm{P}_{\mathrm{W}_{\mathrm{TT}} \mu^{\prime} \nu^{\prime}, \mu \nu}=\frac{1}{4} R^{\mu^{\prime} \mu} R^{\nu^{\prime} \nu}
$$




$$
\begin{aligned}
& \mathrm{P}_{\mathrm{W}_{\mathrm{TL}}}^{\mu^{\prime} \nu^{\prime}, \mu \nu}=\frac{1}{2} R^{\mu^{\prime} \mu} Q_{2}^{\nu} Q_{2}^{\nu^{\prime}}, \\
& \mathrm{P}_{\mathrm{W}_{\mathrm{LT}} \mu^{\prime} \nu^{\prime}, \mu \nu}^{\mu^{\prime}}=\frac{1}{2} Q_{1}^{\mu^{\prime}} Q_{1}^{\mu} R^{\nu^{\prime} \nu} \\
& \mathrm{P}_{\mathrm{W}_{\mathrm{LL}}}^{\mu^{\prime} \nu^{\prime}, \mu \nu}=Q_{1}^{\mu^{\prime}} Q_{1}^{\mu} Q_{2}^{\nu^{\prime}} Q_{2}^{\nu} \text {, } \\
& \mathrm{P}_{\mathrm{W}_{\mathrm{TT}}^{\mu^{\prime} \nu^{\prime}, \mu \nu}}^{\tau}=\frac{1}{4}\left(R^{\mu^{\prime} \nu^{\prime}} R^{\mu \nu}+R^{\mu^{\prime} \nu} R^{\nu^{\prime} \mu}-R^{\mu^{\prime} \mu} R^{\nu^{\prime} \nu}\right),
\end{aligned}
$$

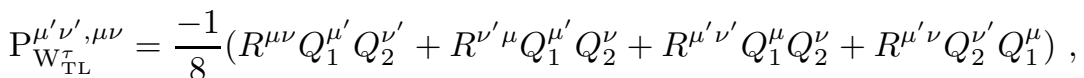

$$
\begin{aligned}
& \mathrm{P}_{\mathrm{W}_{\mathrm{TT}}^{\mu^{\prime} \nu^{\prime}, \mu \nu}}^{\mathrm{a}^{\mathrm{a}}}=\frac{1}{4}\left(R^{\mu^{\prime} \nu^{\prime}} R^{\mu \nu}-R^{\nu^{\prime} \mu} R^{\mu^{\prime} \nu}\right), \\
& \mathrm{P}_{\mathrm{W}_{\mathrm{TL}}^{\mu^{\prime} \nu^{\prime}, \mu \nu}}^{\mathrm{a}}=\frac{-1}{8}\left(R^{\mu \nu} Q_{1}^{\mu^{\prime}} Q_{2}^{\nu^{\prime}}-R^{\nu^{\prime} \mu} Q_{1}^{\mu^{\prime}} Q_{2}^{\nu}+R^{\mu^{\prime} \nu^{\prime}} Q_{1}^{\mu} Q_{2}^{\nu}-R^{\mu^{\prime} \nu} Q_{2}^{\nu^{\prime}} Q_{1}^{\mu}\right) \text {. }
\end{aligned}
$$

\subsection{Derivation of the Cross Section}

Using Eqs. (2.9), (2.10) and (2.11) one obtains by a straightforward (but tedious) calculation

$$
\begin{aligned}
L_{(1)}^{\mu \mu^{\prime}} L_{(2)}^{\nu \nu^{\prime}} W_{\mu^{\prime} \nu^{\prime}, \mu \nu}=Q^{2} P^{2}[ & 4 \rho_{1}^{++} \rho_{2}^{++} W_{\mathrm{TT}}+2\left|\rho_{1}^{+-} \rho_{2}^{+-}\right| W_{\mathrm{TT}}^{\tau} \cos 2 \bar{\phi} \\
& +2 \rho_{1}^{++} \rho_{2}^{00} W_{\mathrm{TL}}+2 \rho_{1}^{00} \rho_{2}^{++} W_{\mathrm{LT}} \\
& \left.+\rho_{1}^{00} \rho_{2}^{00} W_{\mathrm{LL}}-8\left|\rho_{1}^{+0} \rho_{2}^{+0}\right| W_{\mathrm{TL}}^{\tau} \cos \bar{\phi}\right]
\end{aligned}
$$

where $\bar{\phi}$ is the angle between the scattering planes of the $e^{-}$and the $e^{+}$in the center-of-mass system (CMS) of the colliding photons and the $\rho_{\mathrm{i}}$ 's are elements of the photon density matrix:

$$
\begin{aligned}
2 \rho_{1}^{++} & =2 \rho_{1}^{--}=\rho_{1}^{\alpha \beta} R_{\alpha \beta}=\frac{\left(2 p_{1} \cdot p-p \cdot q\right)^{2}}{(p \cdot q)^{2}-Q^{2} P^{2}}+1-4 \frac{m_{\mathrm{e}}^{2}}{Q^{2}}, \\
2 \rho_{2}^{++} & =2 \rho_{2}^{--}=\rho_{2}^{\alpha \beta} R_{\alpha \beta}=\frac{\left(2 p_{2} \cdot q-p \cdot q\right)^{2}}{(p \cdot q)^{2}-Q^{2} P^{2}}+1-4 \frac{m_{\mathrm{e}}^{2}}{P^{2}} \\
\rho_{1}^{00} & =\rho_{1}^{\alpha \beta} Q_{1_{\alpha}} Q_{1 \beta}=2 \rho_{1}^{++}-2+4 \frac{m_{\mathrm{e}}^{2}}{Q^{2}} \\
\rho_{2}^{00} & =\rho_{2}^{\alpha \beta} Q_{2_{\alpha}} Q_{2 \beta}=2 \rho_{2}^{++}-2+4 \frac{m_{\mathrm{e}}^{2}}{P^{2}} \\
2\left|\rho_{1}^{+-} \rho_{2}^{+-}\right| \cos 2 \bar{\phi} & =\frac{C^{2}}{Q^{2} P^{2}}-2\left(\rho_{1}^{++}-1\right)\left(\rho_{2}^{++}-1\right), \\
8\left|\rho_{1}^{+0} \rho_{2}^{+0}\right| \cos \bar{\phi} & =\frac{4 C}{\sqrt{Q^{2} P^{2}}} \frac{\left(2 p_{1} \cdot p-p \cdot q\right)\left(2 p_{2} \cdot q-p \cdot q\right)}{(p \cdot q)^{2}-Q^{2} P^{2}}
\end{aligned}
$$




$$
\text { with } \begin{aligned}
C= & \left(2 p_{1}-q\right)^{\alpha}\left(2 p_{2}-p\right)^{\beta} R_{\alpha \beta}=-\left(2 p_{1}-q\right) \cdot\left(2 p_{2}-p\right) \\
& +\frac{p \cdot q}{(p \cdot q)^{2}-Q^{2} P^{2}}\left(2 p_{1} \cdot p-p \cdot q\right)\left(2 p_{2} \cdot q-p \cdot q\right) \\
\left|\rho_{\mathrm{i}}^{+-}\right|= & \rho_{\mathrm{i}}^{++}-1 \\
\left|\rho_{\mathrm{i}}^{+0}\right|= & \sqrt{\left(\rho_{\mathrm{i}}^{00}+1\right)\left|\rho_{\mathrm{i}}^{+-}\right|} .
\end{aligned}
$$

Note that all these quantities are expressed in terms of the measurable momenta $p_{1}, p_{2}$ and $p_{1}^{\prime}, p_{2}^{\prime}$ (respectively $q, p$ ) only and therefore are entirely known.

With help of Eqs. (2.7) and (2.20) we easily find the fully general final result for the $e e \rightarrow e e X$ cross section $[21,29,2]$ :

$$
\begin{aligned}
d^{6} \sigma(e e \rightarrow e e X)= & \frac{d^{3} p_{1}^{\prime} d^{3} p_{2}^{\prime}}{E_{1}^{\prime} E_{2}^{\prime}} \frac{\alpha^{2}}{16 \pi^{4} Q^{2} P^{2}} \frac{F_{\gamma \gamma}}{F_{e e}}\left[4 \rho_{1}^{++} \rho_{2}^{++} \sigma_{\mathrm{TT}}\right. \\
& +2 \rho_{1}^{00} \rho_{2}^{++} \sigma_{\mathrm{LT}}+2 \rho_{1}^{++} \rho_{2}^{00} \sigma_{\mathrm{TL}}+\rho_{1}^{00} \rho_{2}^{00} \sigma_{\mathrm{LL}} \\
& \left.+2\left|\rho_{1}^{+-} \rho_{2}^{+-}\right| \tau_{\mathrm{TT}} \cos 2 \bar{\phi}-8\left|\rho_{1}^{+0} \rho_{2}^{+0}\right| \tau_{\mathrm{TL}} \cos \bar{\phi}\right]
\end{aligned}
$$

with

$$
\frac{F_{\gamma \gamma}}{F_{e e}}=\left[\frac{(p \cdot q)^{2}-Q^{2} P^{2}}{\left(p_{1} \cdot p_{2}\right)^{2}-m_{e}^{2} m_{e}^{2}}\right]^{1 / 2} .
$$

Here the cross sections $\sigma_{a b}$ (used as a shorthand for $\sigma_{\mathrm{TT}}, \sigma_{\mathrm{TL}}, \sigma_{\mathrm{LT}}, \sigma_{\mathrm{LL}}$, $\left.\tau_{\mathrm{TT}}, \tau_{\mathrm{TL}}, \tau_{\mathrm{TT}}^{\mathrm{a}}, \tau_{\mathrm{TL}}^{\mathrm{a}}\right)$ are identical to the corresponding structure functions $W_{a b}$ up to a division by the appropriate flux factor of the two incoming photons ${ }^{1}$, i.e.

$$
\sigma_{a b}=\frac{1}{2 \sqrt{(p \cdot q)^{2}-Q^{2} P^{2}}} W_{a b}=\frac{1}{2 \nu \bar{\beta}} W_{a b} .
$$

The cross section in (2.22) considerably simplifies in certain kinematical regions $[21,29,2]$. For instance, if both photons are highly virtual Eq. (2.22) can be evaluated in the limit $Q^{2}, P^{2} \gg m_{\mathrm{e}}^{2}[2]$ in which some relations between the elements of the photon density matrix exist. Of special interest is the case where one of the lepton scattering angles becomes small leading to a small virtuality $P^{2} \approx 0$ of the corresponding photon while the other photon provides a hard scale $Q^{2} \gtrsim 1 \mathrm{GeV}^{2}$. In this limit the cross section factorizes into a product of a flux of quasi-real target photons times the cross section for deep inelastic electron-photon scattering, see for example [2]. This process is the classical way of measuring the structure of (quasi-real) photons. The findings in the latter limit can be generalized to the case of photons with non-zero virtuality $P^{2} \neq 0$ as we will see in

\footnotetext{
${ }^{1}$ Note that a factor $\frac{1}{2}$ has already been absorbed into the definition of $W^{\mu^{\prime} \nu^{\prime}, \mu \nu}$ in Eq. (2.8).
} 
Section 4. This allows to study the structure of virtual photons in deep inelastic e $\gamma\left(P^{2}\right)$ scattering processes in a continuous range of the scale $P^{2}$.

\subsection{The Doubly Virtual Box in LO}

In this section we calculate the invariant amplitudes $W_{a b}$ (or the cross sections $\sigma_{a b}$ ) in lowest order perturbation theory. These expressions are usually referred to as 'box' results due to the diagram representing the tensor $W^{\mu^{\prime} \nu^{\prime}, \mu \nu}$, where the photons are attached to a fermion box, see Fig. 4 .

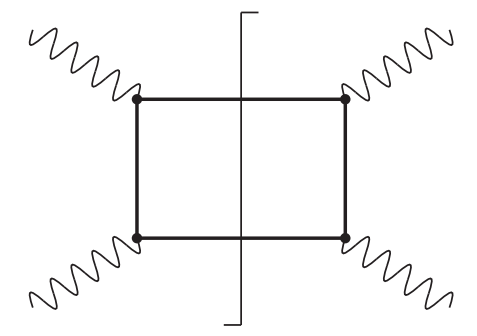

FIG. 4 Box-diagram for $\gamma^{\star}(q, \mu) \gamma^{\star}(p, \nu) \rightarrow \gamma^{\star}\left(q, \mu^{\prime}\right) \gamma^{\star}\left(p, \nu^{\prime}\right)$.There are 4 possibilities to attach the photons to the vertices. (2 for the initial state times 2 for the final state.)

The calculation is a straightforward application of Feynman rules and will only be shortly outlined here. In order to obtain $W^{\mu^{\prime} \nu^{\prime}, \mu \nu}$ according to Eq. (2.8) one has to build up the amplitude $M^{\mu \nu}$ of the process $\gamma^{\star}(q, \mu) \gamma^{\star}(p, \nu) \rightarrow \mathrm{f}\left(k_{1}\right) \overline{\mathrm{f}}\left(k_{2}\right)$ shown in Fig. 5 where $\mathrm{f}$ is either a lepton or a quark of mass $m$. The kinematics can be described with the help

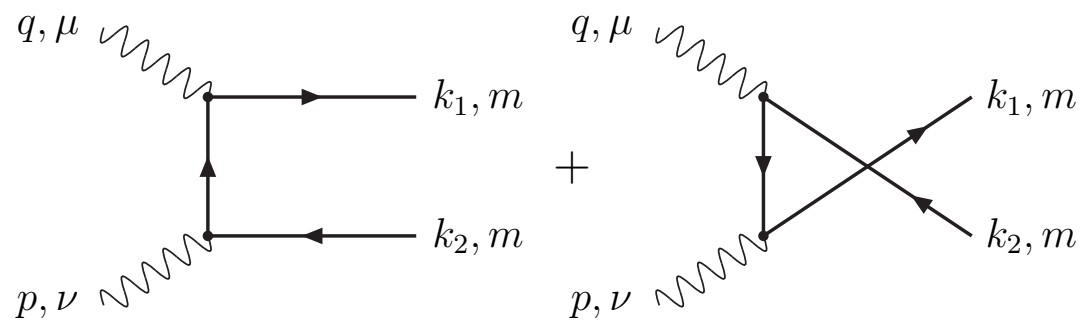

FIG. 5 Amplitude $M^{\mu \nu}$ for the process $\gamma^{\star}(q, \mu) \gamma^{\star}(p, \nu) \rightarrow \mathrm{f}\left(k_{1}\right) \overline{\mathrm{f}}\left(k_{2}\right)$ where $\mathrm{f}$ is a fermion of mass $m$.

of the 4-momentum conservation relation $q+p=k_{1}+k_{2}$ and the usual 
Mandelstam variables

$$
\begin{gathered}
s=(q+p)^{2}, t=\left(q-k_{1}\right)^{2}=\left(p-k_{2}\right)^{2}, u=\left(p-k_{1}\right)^{2}=\left(q-k_{2}\right)^{2}, \\
s_{1} \equiv 2 q \cdot p=s+Q^{2}+P^{2}, t_{1} \equiv t-m^{2}, u_{1} \equiv u-m^{2}
\end{gathered}
$$

satisfying $s_{1}+t_{1}+u_{1}=0$.

The external fermion lines are on-shell, i.e. $k_{1}^{2}=k_{2}^{2}=m^{2}$, whereas the two photons are virtual (space-like): $q^{2}=-Q^{2}<0, p^{2}=-P^{2}<0$. The Dirac traces occurring in $W^{\mu^{\prime} \nu^{\prime}, \mu \nu}$ (due to the closed fermion loop) have been evaluated with the help of the Mathematica [30] package Tracer [31]. Finally, the individual structure functions $W_{a b}$ have been projected out using the projection operators given in Eq. (2.19).

\subsubsection{Unintegrated Structure Functions}

The general structure of the boson-boson fusion cross section (in lowest order) is given by [32, 33]

$$
\frac{d W_{a b}}{d z_{1}}=16 \pi^{2} \alpha^{2} e_{q}^{4} N_{c}\left[\frac{A_{W_{a b}}}{\left(1-z_{1}\right)^{2}}+\frac{B_{W_{a b}}}{z_{1}^{2}}+\frac{C_{W_{a b}}}{1-z_{1}}+\frac{D_{W_{a b}}}{z_{1}}+E_{W_{a b}}\right]
$$

where $z_{1}=1+t_{1} / s_{1}, N_{c}=3$ is the number of colors and $e_{q}$ is the quark charge. The QED case can be obtained from Eq. (2.25) by setting $N_{c} e_{q}^{4} \rightarrow 1$. Note that $z_{1}$ is very similar to the fractional momentum variables generally used to describe energy spectra of heavy quarks (mesons):

$$
z_{1}=\frac{k_{1} \cdot p}{q \cdot p}-\frac{p^{2}}{2 q \cdot p}
$$

On the other hand, $z_{1}=-u_{1} / s_{1}$ (or $1-z_{1}=-t_{1} / s_{1}$ ) can be viewed as a natural dimensionless combination of the Mandelstam variables of the problem. Furthermore, it is convenient to use the dimensionless variables

$$
x=\frac{Q^{2}}{s_{1}}=\frac{Q^{2}}{2 \nu}, \delta=\frac{P^{2}}{s_{1}}=\frac{P^{2}}{2 \nu}, \lambda=\frac{4 m^{2}}{s}
$$

in order to write the coefficients in a form which is manifestly symmetric under $x \leftrightarrow \delta$ (see Sec. 2.2.1) ${ }^{2}$ and which easily allows to read off the important 'massless limits' $P^{2} \rightarrow 0$ or $m^{2} \rightarrow 0$ to be discussed in Appendix A.

With $\bar{\beta}=\sqrt{1-4 x \delta}$ the coefficients read:

$$
\begin{aligned}
A_{W_{\text {Tт }}}= & \frac{-1}{32 \pi \bar{\beta}^{5}}\left[2 x \bar{\beta}^{2}-(1-x-\delta)\left(4 x \delta+\lambda \bar{\beta}^{2}\right)\right]\left[2 \delta \bar{\beta}^{2}\right. \\
& \left.-(1-x-\delta)\left(4 x \delta+\lambda \bar{\beta}^{2}\right)\right]
\end{aligned}
$$

\footnotetext{
${ }^{2}$ Note however that neither $W_{\mathrm{TL}}$ nor $W_{\mathrm{LT}}$ but only the sum $W_{\mathrm{TL}}+W_{\mathrm{LT}}$ is invariant under exchanging $x$ and $\delta$.
} 


$$
\begin{aligned}
& C_{W_{\text {тT }}}=\frac{-1}{16 \pi \bar{\beta}^{5}}\left[\lambda^{2} \bar{\beta}^{4}(1-x-\delta)^{2}-2 \lambda \bar{\beta}^{2}(1-x-\delta)^{2}\right. \\
& -2\left(1-2 x(1-x)-2 \delta(1-\delta)-4 x \delta\left(x^{2}+\delta^{2}\right)\right. \\
& \left.\left.+8 x^{2} \delta^{2}\left[1+(1-x-\delta)^{2}\right]\right)\right] \\
& E_{W_{\mathrm{TT}}}=\frac{-1}{4 \pi \bar{\beta}^{5}}[1-2 x(1-x)-2 \delta(1-\delta)+4 x \delta(1-2 x)(1-2 \delta)] \\
& A_{W_{\mathrm{TL}}}=\frac{\delta(1-x-\delta)}{4 \pi \bar{\beta}^{5}} x\left[2 x \bar{\beta}^{2}-(1-x-\delta)\left(4 x \delta+\lambda \bar{\beta}^{2}\right)\right] \\
& C_{W_{\mathrm{TL}}}=\frac{\delta(1-x-\delta)}{-4 \pi \bar{\beta}^{5}}\left[\lambda \bar{\beta}^{2}(1+2 x(-1+x-\delta))+4 x(-1+2 x\right. \\
& +2 \delta-2 x \delta(1+x+\delta))] \\
& E_{W_{\mathrm{TL}}}=\frac{\delta(1-x-\delta)}{\pi \bar{\beta}^{5}}(1-2 x)^{2} \\
& A_{W_{\mathrm{LL}}}=-\frac{2 x^{2} \delta^{2}(1-x-\delta)^{2}}{\pi \bar{\beta}^{5}} \\
& C_{W_{\mathrm{LL}}}=\frac{2 x \delta(1-x-\delta)^{2}}{\pi \bar{\beta}^{5}}(1+2 x \delta) \\
& E_{W_{\mathrm{LL}}}=-\frac{8 x \delta(1-x-\delta)^{2}}{\pi \bar{\beta}^{5}} \\
& A_{W_{\mathrm{TT}}^{\tau}}=\frac{-1}{32 \pi \bar{\beta}^{5}}(1-x-\delta)^{2}\left[4 x \delta+\lambda \bar{\beta}^{2}\right]^{2} \\
& C_{W_{\mathrm{TT}}^{\tau}}=\frac{-1}{16 \pi \bar{\beta}^{5}}\left[\lambda^{2} \bar{\beta}^{4}(1-x-\delta)^{2}-4 \lambda \bar{\beta}^{2}(1-x-\delta)(-x-\delta\right. \\
& +2 x \delta(1+x+\delta))-x \delta(1-2 x(1-x)-2 \delta(1-\delta) \\
& \left.\left.+2 x \delta\left(1-2 x-2 \delta-x^{2}-\delta^{2}+6 x \delta\right)\right)\right] \\
& E_{W_{\mathrm{TT}}^{\tau}}=\frac{-1}{2 \pi \bar{\beta}^{5}}(x+\delta-4 x \delta)^{2} \\
& A_{W_{\mathrm{TL}}^{\tau}}=\frac{\sqrt{x \delta}(1-x-\delta)}{16 \pi \bar{\beta}^{5}}(1-2 x)(1-2 \delta)\left(4 x \delta+\lambda \bar{\beta}^{2}\right)
\end{aligned}
$$




$$
\begin{aligned}
& C_{W_{\mathrm{TL}}^{\tau}}=\frac{\sqrt{x \delta}(1-x-\delta)}{4 \pi \bar{\beta}^{5}}\left[4 x \delta(-3+x+\delta)+2 x+2 \delta-\lambda \bar{\beta}^{2}(1-x-\delta)\right] \\
& E_{W_{\mathrm{TL}}^{\tau}}=\frac{\sqrt{x \delta}(1-x-\delta)}{2 \pi \bar{\beta}^{5}}(1-4 x-4 \delta+12 x \delta) \\
& A_{W_{\mathrm{TT}}^{\mathrm{a}}}=\frac{1}{16 \pi \bar{\beta}^{3}}\left[-2 x \delta \bar{\beta}^{2}+(1-x-\delta)\left(4 x \delta+\lambda \bar{\beta}^{2}\right)\right] \\
& C_{W_{\mathrm{TT}}^{\mathrm{a}}}=-\frac{(1-2 x)(1-2 \delta)}{8 \pi \bar{\beta}^{3}} \\
& E_{W_{\mathrm{TT}}^{\mathrm{a}}}=\frac{(1-2 x)(1-2 \delta)}{4 \pi \bar{\beta}^{3}} \\
& A_{W_{\mathrm{TL}}^{\mathrm{a}}}=\frac{\sqrt{x \delta}(1-x-\delta)}{16 \pi \bar{\beta}^{3}}\left(-4 x \delta+\lambda \bar{\beta}^{2}\right) \\
& C_{W_{\mathrm{TL}}^{\mathrm{a}}}=\frac{\sqrt{x \delta}(1-x-\delta)}{\pi \bar{\beta}^{3}} x \delta \\
& E_{W_{\mathrm{TL}}^{\mathrm{a}}}=-\frac{\sqrt{x \delta}(1-x-\delta)}{2 \pi \bar{\beta}^{3}} \\
& B_{W_{a b}}=A_{W_{a b}},
\end{aligned}
$$

The coefficients of $W_{\mathrm{LT}}$ can be obtained from the corresponding ones of $W_{\mathrm{TL}}$ by exchanging $x \leftrightarrow \delta: A_{W_{\mathrm{LT}}}=A_{W_{\mathrm{TL}}}[x \leftrightarrow \delta], C_{W_{\mathrm{LT}}}=C_{W_{\mathrm{TL}}}[x \leftrightarrow \delta]$, and $E_{W_{\mathrm{LT}}}=E_{W_{\mathrm{TL}}}[x \leftrightarrow \delta]$.

It is noteworthy that our results in Eq. (2.25) generalize the $z$-differential expressions for the (QED) structure functions $F_{2}^{\gamma}, F_{\mathrm{L}}^{\gamma}$, and $F_{\mathrm{T}}^{\gamma}$ of real photons given in [34] to the $P^{2} \neq 0$ case.

\subsubsection{Inclusive Structure Functions}

The desired inclusive structure functions are obtained by integrating over the kinematically allowed range in $z_{1}$. The boundaries for the $z_{1^{-}}$ integration are given by

$$
z_{1, \pm}=(1 \pm \beta \bar{\beta}) / 2
$$

with $\beta^{2}=1-4 m^{2} / s=1-\lambda$ and $\bar{\beta}^{2}=1-4 x^{2} P^{2} / Q^{2}=1-4 x \delta$.

Noticing that

$$
\begin{gathered}
z_{1,+}-z_{1,-}=\beta \bar{\beta}, \quad 1-z_{1,+}=z_{1,-}, \quad 1-z_{1,-}=z_{1,+}, \\
z_{1,+} z_{1,-}=\left(1-z_{1,+}\right)\left(1-z_{1,-}\right)=\frac{4 x \delta+\lambda \bar{\beta}^{2}}{4}
\end{gathered}
$$


the required integrals can be immediately obtained

$$
\begin{aligned}
\int_{z_{1,-}}^{z_{1,+}} \frac{d z_{1}}{\left(1-z_{1}\right)^{2}} & =\int_{z_{1,-}}^{z_{1,+}} \frac{d z_{1}}{z_{1}^{2}}=\frac{\beta \bar{\beta}}{z_{1,+} z_{1,-}}=\frac{4 \beta \bar{\beta}}{4 x \delta+\lambda \bar{\beta}^{2}} \\
\int_{z_{1,-}}^{z_{1,+}} \frac{d z_{1}}{\left(1-z_{1}\right)} & =\int_{z_{1,-}}^{z_{1,+}} \frac{d z_{1}}{z_{1}}=\ln \frac{z_{1,+}}{z_{1,-}}=\ln \frac{1+\beta \bar{\beta}}{1-\beta \bar{\beta}} \\
\int_{z_{1,-}}^{z_{1,+}} d z_{1} & =\beta \bar{\beta} .
\end{aligned}
$$

Now Eq. (2.25) can be integrated using Eq. (2.37) and we arrive at the following result for the inclusive structure functions expressed by the coefficients given in the previous Section 2.4.1:

$$
W_{a b}=16 \pi^{2} \alpha^{2} e_{q}^{4} N_{c} \Theta\left(\beta^{2}\right)\left\{2 C_{W_{a b}} L+\beta \bar{\beta}\left(2 A_{W_{a b}} \frac{4}{4 x \delta+\lambda \bar{\beta}^{2}}+E_{W_{a b}}\right)\right\}
$$

with

$$
L=\ln \frac{1+\beta \bar{\beta}}{1-\beta \bar{\beta}} .
$$

The $\Theta$-function guarantees that the physical threshold condition $s \geq 4 m^{2}$ is satisfied. It is easy to see that $\beta^{2} \geq 0$ also implies $\bar{\beta}^{2} \geq 0$.

Recalling the relation $\sigma_{a b}=\frac{1}{2 \nu \beta} W_{a b}$ we can rewrite Eq. (2.38) for the photon-photon cross sections

$$
\begin{aligned}
& \sigma_{a b}=N\left\{2 C_{W_{a b}} L+\beta \bar{\beta}\left(2 A_{W_{a b}} \frac{4}{4 x \delta+\lambda \bar{\beta}^{2}}+E_{W_{a b}}\right)\right\} \\
& \text { with } \quad N \equiv \frac{16 \pi^{2} \alpha^{2} N_{c} e_{q}^{4}}{2 \nu \bar{\beta}} \Theta\left(\beta^{2}\right) .
\end{aligned}
$$

Inserting the coefficients given in Eqs. (2.27)-(2.34) into Eq. (2.40) one obtains the final result for the doubly virtual box $\gamma^{\star}\left(Q^{2}\right) \gamma^{\star}\left(P^{2}\right) \rightarrow q \bar{q}$ in leading order:

$$
\begin{aligned}
\sigma_{\mathrm{TT}} & =\frac{N}{4 \pi} \frac{1}{\bar{\beta}^{5}}\left\{\left[1-2 x(1-x)-2 \delta(1-\delta)-4 x \delta\left(x^{2}+\delta^{2}\right)\right.\right. \\
& \left.+8 x^{2} \delta^{2}\left[1+(1-x-\delta)^{2}\right]+\lambda \bar{\beta}^{2}(1-x-\delta)^{2}-\frac{1}{2} \lambda^{2} \bar{\beta}^{4}(1-x-\delta)^{2}\right] L \\
& +\beta \bar{\beta}\left[4 x(1-x)-1+4 \delta(1-\delta)-8 x \delta\left(1-x^{2}-\delta^{2}\right)\right. \\
& \left.\left.-\left(4 x \delta+\lambda \bar{\beta}^{2}\right)(1-x-\delta)^{2}-\frac{4 x \delta \bar{\beta}^{4}}{4 x \delta+\lambda \bar{\beta}^{2}}\right]\right\}
\end{aligned}
$$




$$
\begin{aligned}
& \sigma_{\mathrm{LT}}=\frac{N}{4 \pi} \frac{4}{\bar{\beta}^{5}}(1-x-\delta)\left\{x \left[-\frac{1}{2} \lambda \bar{\beta}^{2}(1-2 \delta(1+x-\delta))\right.\right. \\
&-2 \delta(-1+2 x+2 \delta-2 x \delta(1+x+\delta))] L \\
&\left.+\beta \bar{\beta}\left[x\left(1-6 \delta+6 \delta^{2}+2 x \delta\right)+\delta \bar{\beta}^{2} \frac{4 x \delta}{4 x \delta+\lambda \bar{\beta}^{2}}\right]\right\} \\
& \sigma_{\mathrm{TL}}=\sigma_{\mathrm{LT}}[x \leftrightarrow \delta] \\
& \sigma_{\mathrm{LL}}=\frac{N}{4 \pi} \frac{16}{\bar{\beta}^{5}} x \delta(1-x-\delta)^{2}\left\{(1+2 x \delta) L-2 \beta \bar{\beta} \frac{6 x \delta+\lambda \bar{\beta}^{2}}{4 x \delta+\lambda \bar{\beta}^{2}}\right\} \\
& \tau_{\mathrm{TT}}=\frac{N}{4 \pi} \frac{1}{\bar{\beta}^{5}}\left\{\left[\frac{1}{2} x \delta(1-2 x(1-x)-2 \delta(1-\delta)+2 x \delta(1-2 x-2 \delta\right.\right. \\
&\left.\left.-x^{2}-\delta^{2}+6 x \delta\right)\right)-2 \lambda \bar{\beta}^{2}(1-x-\delta)(x+\delta-2 x \delta(1+x+\delta)) \\
&\left.-\frac{1}{2} \lambda^{2} \bar{\beta}^{4}(1-x-\delta)^{2}\right] L-\beta \bar{\beta}\left[(1-x-\delta)^{2}\left(4 x \delta+\lambda \bar{\beta}^{2}\right)\right. \\
&\left.\left.+2(x+\delta-4 x \delta)^{2}\right]\right\} \\
& \tau_{\mathrm{TL}}^{\mathrm{a}}=\frac{N}{4 \pi} \frac{4}{\bar{\beta}^{3}} \sqrt{x \delta}(1-x-\delta)\left\{2 x \delta L-\beta \bar{\beta} \frac{4 x \delta}{4 x \delta+\lambda \bar{\beta}^{2}}\right\} \\
& \tau_{\mathrm{TL}}=\frac{N}{4 \pi} \frac{2}{\bar{\beta}^{5}} \sqrt{x \delta}(1-x-\delta)\{[2 x+2 \delta-4 x \delta(3-x-\delta) \\
&\left.\left.-\lambda \bar{\beta}^{2}(1-x-\delta)\right] L+\beta \bar{\beta}(1-3 x-3 \delta+8 x \delta)\right\} \\
&\left.\bar{\beta}^{3}(2 x-1)(1-2 \delta) L+\beta \bar{\beta}\left[\frac{-4 x \delta \bar{\beta}^{2}}{4 x \delta+\lambda \bar{\beta}^{2}}+3-4 x-4 \delta+4 x \delta\right]\right\} \\
& \\
&
\end{aligned}
$$

This re-calculation is in agreement with the results of Ref. [21] (with $N_{c} e_{q}^{4} \rightarrow 1$ ) with exception of a relative sign between the part contain- 
ing the logarithm $L$ and the part proportional to $\beta \bar{\beta}$ in $\tau_{\mathrm{TL}}^{\mathrm{a}}$. This relative sign has also been noted in [18] where in addition the overall sign in $\tau_{\mathrm{TL}}$ is different. Concerning the latter, our calculation agrees with the results of [21].

\subsubsection{Discussion of the results}

- The general structure of the final results in (2.41)-(2.48) is given by $\sigma_{a b}(x, \delta, \lambda)=\sigma_{1, a b}(x, \delta, \lambda) L+\beta \bar{\beta} \sigma_{2, a b}(x, \delta, \lambda)$ as is evident from Eq. (2.40). The logarithm $L$ stems from the $1 / z_{1}$ and $1 /\left(1-z_{1}\right)$ terms in Eq. (2.25). It develops a mass singularity (collinear singularity) in the limit of small quark masses $m$ and small photon virtualities $P^{2}$. This is related to the fact that the upper and lower integration bounds in Eq. (2.35) approach one and zero in this limit: $z_{1,+} \rightarrow 1$, $z_{1,-} \rightarrow 0$. As long as $m$ or $P^{2}$ is kept non-zero the singularity is regularized. On the other hand, the $1 / z_{1}^{2}$ (and $\left.1 /\left(1-z_{1}\right)^{2}\right)$ parts give finite contributions because the coefficients $A_{W_{a b}}=B_{W_{a b}}$ in Eqs. (2.27)-(2.34) vanish for $P^{2}, m \rightarrow 0$.

- The photon photon cross sections $\sigma_{\mathrm{TT}}, \sigma_{\mathrm{LL}}, \tau_{\mathrm{TT}}, \tau_{\mathrm{TL}}, \tau_{\mathrm{TT}}^{\mathrm{a}}$ and $\tau_{\mathrm{TL}}^{\mathrm{a}}$ in $(2.41)-(2.48)$ are symmetric w.r.t. $x \leftrightarrow \delta$ whereas $\sigma_{\mathrm{TL}}(x, \delta)=$ $\sigma_{\mathrm{LT}}(\delta, x)$. This is a direct consequence of the symmetries of the helicity amplitudes as has been discussed in Sec. 2.2.1.

- As a rule each longitudinal polarisation vector results in a factor $\sqrt{P^{2}}$ or $\sqrt{Q^{2}}$ depending on which of the photons, $\gamma\left(P^{2}\right)$ or $\gamma\left(Q^{2}\right)$, is longitudinal. (It is helpful to recall that in the helicity amplitudes $W_{m^{\prime} n^{\prime}, m n}$ the helicities $n^{\prime}$ and $n$ refer to $\gamma\left(P^{2}\right)$, whereas $m^{\prime}$ and $m$ refer to $\gamma\left(Q^{2}\right)$.) These factors guarantee that the contributions from longitudinal photons vanish - with one exception which is discussed in App. A- in the limit when these photons become real. This rule explains several prefactors $x, \delta, \sqrt{x}$ and $\sqrt{\delta}$ in Eqs. (2.27)-(2.34) and (2.41)-(2.48).

- Various limits can be easily derived from the general expressions in (2.41)-(2.48), e.g.:

$-m^{2}, P^{2} \ll Q^{2}$

- $m^{2}=0$ and $m^{2}=0, P^{2} \ll Q^{2}$

$-P^{2}=0$ and $P^{2}=0, m^{2} \ll Q^{2}$

A useful compilation of some of these limits of the doubly virtual box expressions can be found in App. A. 


\subsubsection{Applications}

The doubly virtual box expressions have several applications. First of all, they can be applied in the QED case (mainly $\gamma^{\star}\left(Q^{2}\right) \gamma^{\star}\left(P^{2}\right) \rightarrow \mu^{+} \mu^{-}$) irrespective of $P^{2}$ and $Q^{2}$ as long as $Q^{2} \geq P^{2}$ is not too large, such that $Z$ or $W$-boson exchange can be neglected. Indeed, the precise measurements of the QED structure of the photon at LEP, see e.g. chapter 6 in [2], nicely agree with the perturbative QED box predictions.

In the QCD case $\left(\gamma^{\star}\left(Q^{2}\right) \gamma^{\star}\left(P^{2}\right) \rightarrow q \bar{q}\right)$ one can distinguish different 'phases' depending on the virtualities of the two photons, $Q^{2}$ and $P^{2}$, and on the invariant photon-photon mass $W^{2}=(p+q)^{2}$. These scales are in comparison with a typical hadronic scale like the mass of the rho meson $m_{\rho}=770 \mathrm{MeV}$ or $\Lambda_{\mathrm{QCD}}=\mathcal{O}(200 \mathrm{MeV})$. Anyhow, the uncertainty in choosing the appropriate hadronic scale can be absorbed into the ignorance of what ' $\gg$ ' or ' $\ll$ ' exactly means such that we can take $\Lambda_{\mathrm{QCD}}$ as our hadronic scale in the following examples:

- $Q^{2}, P^{2} \gg \Lambda_{\mathrm{QCD}}^{2}$ :

In this region of phase space we have a purely perturbative ('golden') process within QCD where the predictions of Eqs. (2.41)-(2.48) are generally expected to be applicable. We can further subdivide:

- $Q^{2} \simeq P^{2} \gg \Lambda_{\mathrm{QCD}}^{2}$

$-Q^{2} \gg P^{2} \gg \Lambda_{\mathrm{QCD}}^{2}$ :

In this case we can think of a virtual target photon $\gamma\left(P^{2}\right)$ whose structure is resolved by a probe photon $\gamma\left(Q^{2}\right)$. Since $P^{2} \gg \Lambda_{\mathrm{QCD}}^{2}$ the photon structure functions are perturbatively calculable either in FOPT or by resumming potentially large logarithms $\ln Q^{2} / P^{2}$ occuring in the fixed order box calculation to all orders via renormalization group (RG) techniques [35]. This can be achieved by solving the Altarelli-Parisi evolution equations for the photon supplemented with the appropriate (perturbative) boundary conditions $[16,15]$ which can be inferred from the fixed order box calculation [36].

- $W^{2} \gg Q^{2}, P^{2} \gg \Lambda_{\mathrm{QCD}}^{2}$ :

The high energy limit of virtual photon photon collisions has attracted a lot of interest in the literature [37, 38, 39] since it might signal BFKL [40, 41] dynamics. From this point of view the quark box results in Eqs. (2.41)-(2.48) constitute a background. On the other hand they can also be viewed as a competitive theoretical approach to experimental measurements $[42,43]$. Recently, $\mathcal{O}\left(\alpha_{s}\right)$ corrections to the effective (measurable) combination $\sigma_{\text {eff }}=\sigma_{\mathrm{TT}}+\varepsilon_{1} \sigma_{\mathrm{TL}}+\varepsilon_{2} \sigma_{\mathrm{LT}}+\varepsilon_{1} \varepsilon_{2} \sigma_{\mathrm{LL}}$ with $\varepsilon_{i}=2\left(1-y_{i}\right) /\left(1+\left(1-y_{i}\right)^{2}\right)$ have been obtained in Ref. [44]. For an overview of the current status see, e.g., Fig. 1 in [45]. 
- $Q^{2} \gg \Lambda_{\mathrm{QCD}}^{2} \gtrsim P^{2}$ :

If one of the photons is (quasi-)real or slightly virtual perturbation theory is clearly not reliable due to non-perturbative 'long distance' effects which are cut off in the previous cases by the large virtuality $P^{2}$. For this reason the perturbative box calculation is in principle not directly applicable. Nevertheless, first analyses of real photon structure functions have been performed within the naive quarkparton model (QPM) [46] where the so-called 'QPM' or 'Box' expressions for the photon structure functions have been calculated according to the process $\gamma^{\star}\left(Q^{2}\right) \gamma \rightarrow q \bar{q}$ employing a finite quark mass $m=\mathcal{O}(300 \mathrm{MeV})$. Of course, this procedure to regularize the mass singularities by some quark masses is ad hoc: the quark masses are unphysical and, moreover, the long-distance dynamics is certainly not well described by (massive) free quark propagators. Therefore, in modern approaches to the structure of (quasi-)real and (slightly) virtual target photons $\gamma\left(P^{2}\right)[8,15,6]$ the photon structure functions (or the photon tensor) are factorized [47] into non-perturbative parton distribution functions to be fixed by experimental information and calculable short distance (Wilson) coefficient functions.

\section{PHOTON STRUCTURE FUNCTIONS}

It is the aim of this section to define structure functions of a (virtual) target photon and to relate them to the invariant functions $W_{a b}$. The defining relations will be generally valid for arbitrary $P^{2}$. However, they only have a meaningful interpretation as structure functions of a target photon probed by a deeply virtual photon $\gamma\left(Q^{2}\right)$ in the limit $P^{2} \ll Q^{2}$.

The structure tensor for time-like [48] and space-like [49] spin-1 targets is described by 8 structure functions (as long as e.m. interactions are considered). Indeed, in Ref. [49] the case of a (space-like) virtual target photon has been considered. However, for the discussion below (involving transversely and longitudinally polarized targets) it is necessary to start with the 4-th rank photon tensor given in Eq. (2.11). It is possible to obtain the 2-nd rank structure tensor by contracting the (4-th rank) photon tensor with the polarization vectors of the target photons:

$$
W_{\mu^{\prime} \mu}\left(q, p, \lambda, \lambda^{\prime}\right) \equiv \epsilon_{\lambda^{\prime}}^{\star \nu^{\prime}}(p) \epsilon_{\lambda}^{\nu}(p) W_{\mu^{\prime} \nu^{\prime}, \mu \nu} .
$$

The spin-1 structure tensor can be decomposed into a spin-averaged, a spin-dependent symmetric (singly-polarized) and a spin-dependent antisymmetric part $[48,49]$. This can be achieved by writing the polarization vectors in the following manner:

$$
E^{\nu^{\prime}} F^{\nu}=-\frac{1}{2} g^{\nu^{\prime} \nu}+\frac{1}{2}\left(E^{\nu^{\prime}} F^{\nu}+F^{\nu^{\prime}} E^{\nu}+g^{\nu^{\prime} \nu}\right)+\frac{1}{2}\left(E^{\nu^{\prime}} F^{\nu}-F^{\nu^{\prime}} E^{\nu}\right)
$$


with $E^{\nu^{\prime}}=\epsilon_{\lambda^{\prime}}^{\star \nu^{\prime}}(p)$ and $F^{\nu}=\epsilon_{\lambda}^{\nu}(p)$. The spin-averaged (structure functions $F_{1}, F_{2}$ ) and spin-dependent antisymmetric parts (structure functions $g_{1}$, $\left.g_{2}\right)$ are well-known from spin-1/2 targets. On the other hand the spindependent symmetric tensor occurs in the spin- 1 case for the first time. It can be described in terms of 4 structure functions $b_{1}, \ldots, b_{4}$ [48].

In the rest of this section we discuss the spin-averaged part in more detail. In view of the recent literature on the parton content of virtual longitudinal photons $[22,23]$ and the factorization to be addressed in Sec. 4 we discuss also structure functions for transversely and longitudinally polarized targets. The following expressions are simplified if one introduces the transverse components of a four-vector $x_{\mu}$ and of the metric tensor $g_{\mu \nu}$

$$
x_{\mu}^{T}=x_{\mu}-\frac{q \cdot x}{q^{2}} q_{\mu}, \quad g_{\mu \nu}^{T}=g_{\mu \nu}-\frac{1}{q^{2}} q_{\mu} q_{\nu},
$$

where 'transverse' refers to $q: q \cdot x^{T}=0, q^{\mu} g_{\mu \nu}^{T}=q^{\nu} g_{\mu \nu}^{T}=0$.

\subsection{Structure Functions for a Spin-Averaged Photon}

Usually one introduces structure functions for a spin-averaged target photon. The corresponding structure tensor can be obtained by contracting $W_{\mu^{\prime} \nu^{\prime}, \mu \nu}$ given in Eq. (2.11) with the metric tensor $g^{\nu \nu^{\prime}}$. With the help of Eqs. (2.12) and (2.18) one obtains ${ }^{3}$ :

$$
\begin{aligned}
W_{\mu^{\prime} \mu}^{<\gamma>} & \equiv \frac{-g^{\nu \nu^{\prime}}}{2} W_{\mu^{\prime} \nu^{\prime}, \mu \nu} \\
& =R_{\mu^{\prime} \mu}\left[W_{\mathrm{TT}}-\frac{1}{2} W_{\mathrm{TL}}\right]+Q_{1 \mu^{\prime}} Q_{1 \mu}\left[W_{\mathrm{LT}}-\frac{1}{2} W_{\mathrm{LL}}\right] \\
& =-g_{\mu^{\prime} \mu}^{T}\left[W_{\mathrm{TT}}-\frac{1}{2} W_{\mathrm{TL}}\right]+p_{\mu^{\prime}}^{T} p_{\mu}^{T} \frac{Q^{2}}{\nu^{2} \bar{\beta}^{2}}\left[W_{2 \mathrm{~T}}-\frac{1}{2} W_{2 \mathrm{~L}}\right]
\end{aligned}
$$

where $W_{2 \mathrm{~T}} \equiv W_{\mathrm{TT}}+W_{\mathrm{LT}}$ and $W_{2 \mathrm{~L}} \equiv W_{\mathrm{TL}}+W_{\mathrm{LL}}$. Recall that the first index $(a=2, \mathrm{~T}, \mathrm{~L})$ of the invariant functions $W_{a b}$ refers to the probe photon and the second one $(b=\mathrm{T}, \mathrm{L})$ to the target photon.

Alternatively the spin-averaged tensor can be expressed in standard form in terms of the structure functions $F_{1} \equiv W_{1}$ and $F_{2} \equiv \nu W_{2}$ :

$$
\frac{1}{8 \pi^{2} \alpha} W_{\mu^{\prime} \mu}^{<\gamma>}=-g_{\mu^{\prime} \mu}^{T} F_{1}^{<\gamma>}+p_{\mu^{\prime}}^{T} p_{\mu}^{T} \frac{1}{\nu} F_{2}^{<\gamma>} .
$$

Comparing Eqs. (3.52) and (3.53) we find

$$
\begin{aligned}
2 x F_{1}^{<\gamma>} & =\frac{1}{8 \pi^{2} \alpha} \frac{Q^{2}}{\nu}\left[W_{\mathrm{TT}}-\frac{1}{2} W_{\mathrm{TL}}\right] \\
F_{2}^{<\gamma>} & =\frac{1}{8 \pi^{2} \alpha} \frac{Q^{2}}{\nu} \frac{1}{\bar{\beta}^{2}}\left[W_{2 \mathrm{~T}}-\frac{1}{2} W_{2 \mathrm{~L}}\right] .
\end{aligned}
$$

\footnotetext{
${ }^{3}$ Of course the virtual photon has three $(+,-, 0)$ degrees of freedom. The factor $1 / 2$ guarantees the conventional normalization in the real photon limit with only two $(+,-)$ transverse degrees of freedom.
} 
These relations can be re-expressed in terms of the photon-photon cross sections $\sigma_{a b}=W_{a b} /(2 \nu \bar{\beta})(a, b=2, \mathrm{~L}, \mathrm{~T})[2,21,29]$

$$
\begin{aligned}
2 x F_{1}^{<\gamma>} & =\frac{Q^{2}}{4 \pi^{2} \alpha} \bar{\beta}\left[\sigma_{\mathrm{TT}}-\frac{1}{2} \sigma_{\mathrm{TL}}\right] \\
F_{2}^{<\gamma>} & =\frac{Q^{2}}{4 \pi^{2} \alpha} \frac{1}{\bar{\beta}}\left[\sigma_{2 \mathrm{~T}}-\frac{1}{2} \sigma_{2 \mathrm{~L}}\right] .
\end{aligned}
$$

Finally, $F_{\mathrm{L}}$ satisfies the usual relation

$$
F_{\mathrm{L}}^{<\gamma>}=\bar{\beta}^{2} F_{2}^{<\gamma>}-2 x F_{1}^{<\gamma>} .
$$

This can be seen by contracting $W_{\mu^{\prime} \mu}^{<\gamma>}$ with the polarization vectors of longitudinal probe photons given in Eq. (2.15) thereby employing again the orthogonality relations in Eq. (2.18)

$$
\begin{aligned}
W_{\mathrm{L}}^{<\gamma>} & \equiv \epsilon_{0}^{\star \mu^{\prime}}(q) \epsilon_{0}^{\mu}(q) W_{\mu^{\prime} \mu}^{<\gamma>}=Q_{1}^{\mu^{\prime}} Q_{1}^{\mu} W_{\mu^{\prime} \mu}^{<\gamma>}=W_{\mathrm{LT}}-\frac{1}{2} W_{\mathrm{LL}} \\
& =8 \pi^{2} \alpha\left[-F_{1}^{<\gamma>}+\frac{\bar{\beta}^{2}}{2 x} F_{2}^{<\gamma>}\right]
\end{aligned}
$$

followed by the appropriate normalization:

$$
F_{\mathrm{L}}^{<\gamma>}=\frac{1}{8 \pi^{2} \alpha} 2 x W_{\mathrm{L}}^{<\gamma>} .
$$

\subsection{Longitudinal and Transverse Target Photons}

Since the fluxes of transverse and longitudinal virtual photons will turn out to be different (see Eq. (4.67) below) it is most convenient to introduce structure functions of transverse respectively longitudinal target photons (instead of spin-averaged target photons). The procedure is completely analogous to the one in the previous section using again Eqs. (2.15)-(2.18) and for this reason the description will be brief.

I. Transverse Photons

With the help of Eq. (2.15) we can construct the structure tensor for a transverse photon target which can can be cast again into different forms

$$
\begin{aligned}
W_{\mu^{\prime} \mu}^{\gamma_{\mathrm{T}}} & \equiv \frac{1}{2}\left[\epsilon_{+}^{\star \nu^{\prime}}(p) \epsilon_{+}^{\nu}(p)+\epsilon_{-}^{\star \nu^{\prime}}(p) \epsilon_{-}^{\nu}(p)\right] W_{\mu^{\prime} \nu^{\prime}, \mu \nu}=\frac{1}{2} R^{\nu^{\prime} \nu} W_{\mu^{\prime} \nu^{\prime}, \mu \nu} \\
& =R_{\mu^{\prime} \mu} W_{\mathrm{TT}}+Q_{1 \mu^{\prime}} Q_{1 \mu} W_{\mathrm{LT}}=-g_{\mu^{\prime} \mu}^{T} W_{\mathrm{TT}}+p_{\mu^{\prime}}^{T} p_{\mu}^{T} \frac{Q^{2}}{\nu^{2} \bar{\beta}^{2}} W_{2 \mathrm{~T}} \\
& \stackrel{!}{=} 8 \pi^{2} \alpha\left[-g_{\mu^{\prime} \mu}^{T} F_{1}^{\gamma_{\mathrm{T}}}+p_{\mu^{\prime}}^{T} p_{\mu}^{T} \frac{1}{\nu} F_{2}^{\gamma_{\mathrm{T}}}\right]
\end{aligned}
$$

and we can directly read off the structure functions:

$$
\begin{aligned}
2 x F_{1}^{\gamma_{\mathrm{T}}} & =\frac{1}{8 \pi^{2} \alpha} \frac{Q^{2}}{\nu} W_{\mathrm{TT}}=\frac{Q^{2}}{4 \pi^{2} \alpha} \bar{\beta} \sigma_{\mathrm{TT}} \\
F_{2}^{\gamma_{\mathrm{T}}} & =\frac{1}{8 \pi^{2} \alpha} \frac{Q^{2}}{\nu} \frac{1}{\bar{\beta}^{2}} W_{2 \mathrm{~T}}=\frac{Q^{2}}{4 \pi^{2} \alpha} \frac{1}{\bar{\beta}} \sigma_{2 \mathrm{~T}} .
\end{aligned}
$$


Repeating the steps in Sec. 3.1 to determine $F_{\mathrm{L}}^{<\gamma>}$, we obtain $W_{\mathrm{L}}^{\gamma_{\mathrm{T}}}=W_{\mathrm{LT}}$ implying

$$
F_{\mathrm{L}}^{\gamma_{\mathrm{T}}}=\frac{1}{8 \pi^{2} \alpha} 2 x W_{\mathrm{L}}^{\gamma_{\mathrm{T}}}=\bar{\beta}^{2} F_{2}^{\gamma_{\mathrm{T}}}-2 x F_{1}^{\gamma_{\mathrm{T}}} .
$$

II. Longitudinal Photons

The structure tensor for a longitudinal photon target is given by (using Eq. (2.15))

$$
\begin{aligned}
W_{\mu^{\prime} \mu}^{\gamma_{\mathrm{L}}} & \equiv \epsilon_{0}^{\star \nu^{\prime}}(p) \epsilon_{0}^{\nu}(p) W_{\mu^{\prime} \nu^{\prime}, \mu \nu}=Q_{2}^{\nu^{\prime}} Q_{2}^{\nu} W_{\mu^{\prime} \nu^{\prime}, \mu \nu} \\
& =R_{\mu^{\prime} \mu} W_{\mathrm{TL}}+Q_{1 \mu^{\prime}} Q_{1 \mu} W_{\mathrm{LL}}=-g_{\mu^{\prime} \mu}^{T} W_{\mathrm{TL}}+p_{\mu^{\prime}}^{T} p_{\mu}^{T} \frac{Q^{2}}{\nu^{2} \bar{\beta}^{2}} W_{2 \mathrm{~L}} \\
& \stackrel{!}{=} 8 \pi^{2} \alpha\left[-g_{\mu^{\prime} \mu}^{T} F_{1}^{\gamma_{\mathrm{L}}}+p_{\mu^{\prime}}^{T} p_{\mu}^{T} \frac{1}{\nu} F_{2}^{\gamma_{\mathrm{L}}}\right]
\end{aligned}
$$

and we find the following result for a longitudinal target photon:

$$
\begin{aligned}
2 x F_{1}^{\gamma_{\mathrm{L}}} & =\frac{1}{8 \pi^{2} \alpha} \frac{Q^{2}}{\nu} W_{\mathrm{TL}}=\frac{Q^{2}}{4 \pi^{2} \alpha} \bar{\beta} \sigma_{\mathrm{TL}} \\
F_{2}^{\gamma_{\mathrm{L}}} & =\frac{1}{8 \pi^{2} \alpha} \frac{Q^{2}}{\nu} \frac{1}{\bar{\beta}^{2}} W_{2 \mathrm{~L}}=\frac{Q^{2}}{4 \pi^{2} \alpha} \frac{1}{\bar{\beta}} \sigma_{2 \mathrm{~L}} .
\end{aligned}
$$

Finally, we have (as could be expected)

$$
W_{\mathrm{L}}^{\gamma_{\mathrm{L}}}=W_{\mathrm{LL}} \quad \Rightarrow \quad F_{\mathrm{L}}^{\gamma_{\mathrm{L}}}=\frac{1}{8 \pi^{2} \alpha} 2 x W_{\mathrm{L}}^{\gamma_{\mathrm{L}}}=\bar{\beta}^{2} F_{2}^{\gamma_{\mathrm{L}}}-2 x F_{1}^{\gamma_{\mathrm{L}}} .
$$

Further inspection of Eqs. (3.52)-(3.62) reveals a relation $["<\gamma>=$ $\gamma_{\mathrm{T}}-\frac{1}{2} \gamma_{\mathrm{L}}$ "] between the spin-averaged, transverse and longitudinal target photons

$$
\begin{aligned}
W_{\mu^{\prime} \mu}^{<\gamma>} & =W_{\mu^{\prime} \mu}^{\gamma_{\mathrm{T}}}-\frac{1}{2} W_{\mu^{\prime} \mu}^{\gamma_{\mathrm{L}}} \\
F_{i}^{<\gamma>} & =F_{i}^{\gamma_{\mathrm{T}}}-\frac{1}{2} F_{i}^{\gamma_{\mathrm{L}}} \quad(i=1,2, \mathrm{~L})
\end{aligned}
$$

which is a consequence of the completeness relation for space-like photons (cf. [21], Eq. (B.1)):

$$
\underbrace{\epsilon_{+}^{\star \mu}(p) \epsilon_{+}^{\nu}(p)+\epsilon_{-}^{\star \mu}(p) \epsilon_{-}^{\nu}(p)}_{2 \gamma_{\mathrm{T}}}-\underbrace{\epsilon_{0}^{\star \mu}(p) \epsilon_{0}^{\nu}(p)}_{-\gamma_{\mathrm{L}}}=\underbrace{-g^{\mu \nu}+\frac{p^{\mu} p^{\nu}}{p^{2}}}_{2<\gamma>} .
$$




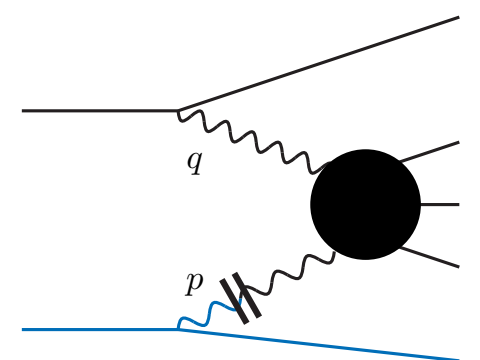

FIG. 6 Factorization of the $e^{+} e^{-} \rightarrow e^{+} e^{-} \gamma^{\star} \gamma^{\star} \rightarrow e^{+} e^{-} X$ cross section into a flux of "target" photons radiated off the lower lepton line times the cross section for deep inelastic electron-photon scattering (black part). The cut in the photon line indicates a time order between the two subprocesses (photon emission followed by deep inelastic e $\gamma$ scattering) and implies also that these two factors are independent of each other.

\section{QED-FACTORIZATION}

It is well known that for $P^{2} \approx 0$ the general cross section for the process $e^{+} e^{-} \rightarrow e^{+} e^{-} \gamma^{\star} \gamma^{\star} \rightarrow e^{+} e^{-} X$ factorizes into a product of a flux of target photons (radiated off the electron) with the deep inelastic electron-photon scattering cross section [29, 2], see Fig. 6 for a graphical representation:

$$
\frac{d \sigma(e e \rightarrow e e X)}{d x d Q^{2} d z d P^{2}}=f_{\gamma_{T} / e}\left(z, P^{2}\right) \frac{d \sigma(e \gamma \rightarrow e X)}{d x d Q^{2}} \quad\left(P^{2} \approx 0\right)
$$

where $z=E_{\gamma} / E \approx y_{2}$ is the fraction of the lepton energy carried by the photon (in the $e^{+} e^{-}$-CMS). The cross section for deep inelastic electronphoton scattering in Eq. (4.65) reads

$$
\begin{aligned}
\frac{d \sigma(e \gamma \rightarrow e X)}{d x d Q^{2}} & =\frac{4 \pi^{2} \alpha y}{x Q^{2}} \frac{\alpha}{2 \pi}\left[\frac{1+(1-y)^{2}}{y} \frac{1}{Q^{2}}\right]\left[2 x F_{1}^{\gamma}+\frac{2(1-y)}{1+(1-y)^{2}} F_{\mathrm{L}}^{\gamma}\right] \\
& =\frac{2 \pi \alpha^{2}}{x Q^{4}}\left[\left(1+(1-y)^{2}\right) 2 x F_{1}^{\gamma}+2(1-y) F_{\mathrm{L}}^{\gamma}\right]
\end{aligned}
$$

with the usual variable $y=\frac{p \cdot q}{p \cdot p_{1}}=y_{1}$. Furthermore, $f_{\gamma_{T} / e}$ denotes the flux factor of transversely (or circularly) polarized photons with virtuality $P^{2}$. For use below we also provide the flux factor $f_{\gamma_{L} / e}$ of longitudinally 
polarized photons:

$$
\begin{aligned}
& f_{\gamma_{T} / e}\left(z, P^{2}\right)=\frac{\alpha}{2 \pi}\left[\frac{1+(1-z)^{2}}{z} \frac{1}{P^{2}}-\frac{2 m_{\mathrm{e}}^{2} z}{P^{4}}\right] \\
& f_{\gamma_{L} / e}\left(z, P^{2}\right)=\frac{\alpha}{2 \pi}\left[\frac{2(1-z)}{z} \frac{1}{P^{2}}\right] .
\end{aligned}
$$

The factorization in (4.65) is essential for relating the concept of the structure of a (real) photon to experimental measurements of two-photon processes. For this reason we want to generalize Eq. (4.65) for photons with virtuality $P^{2} \neq 0$ and show that, in the Bjorken limit, factorization holds for virtual "target" photons as well. For definiteness, as the Bjorken limit we consider

$$
Q^{2} \equiv Q_{1}^{2} \rightarrow \infty, \nu \rightarrow \infty, x=Q^{2} / 2 \nu=\text { fixed } .
$$

Practically, this means $P^{2} \equiv Q_{2}^{2} \ll Q^{2}, \nu$ such that $\delta \equiv P^{2} / 2 \nu=x P^{2} / Q^{2}$ is a small quantity which can be neglected.

The starting point is the general cross section in Eq. (2.22). Employing Eqs. (3.58), (3.59), (3.61), and (3.62) and rearranging the terms inside the square brackets it can be written as

$$
\begin{aligned}
& d^{6} \sigma=\frac{d^{3} p_{1}^{\prime} d^{3} p_{2}^{\prime}}{E_{1}^{\prime} E_{2}^{\prime}} \frac{\alpha^{2}}{16 \pi^{4} Q^{2} P^{2}} \frac{F_{\gamma \gamma}}{F_{e e}}\left[\frac{4 \pi^{2} \alpha}{Q^{2} \bar{\beta}} \times\right. \\
& \left(2 \rho_{2}^{++} 2 \rho_{1}^{++}\left[2 x F_{1}^{\gamma_{\mathrm{T}}}+\varepsilon F_{\mathrm{L}}^{\gamma_{\mathrm{T}}}\right]+\rho_{2}^{00} 2 \rho_{1}^{++}\left[2 x F_{1}^{\gamma_{\mathrm{L}}}+\varepsilon F_{\mathrm{L}}^{\gamma_{\mathrm{L}}}\right]\right) \\
& \left.\quad+2\left|\rho_{1}^{+-} \rho_{2}^{+-}\right| \tau_{\mathrm{TT}} \cos 2 \bar{\phi}-8\left|\rho_{1}^{+0} \rho_{2}^{+0}\right| \tau_{\mathrm{TL}} \cos \bar{\phi}\right] \\
& \text { with } \quad \varepsilon=\frac{\rho_{1}^{00}}{2 \rho_{1}^{++}} .
\end{aligned}
$$

The general strategy will be to demonstrate that $\rho_{2}^{++}$and $\rho_{2}^{00}$ are proportional to the flux factors of transverse and longitudinal photons, respectively, radiated off an electron and that the interference terms disappear after having performed an appropriate angular integration.

In the Bjorken limit it is useful to perform a "light cone decomposition" of the 4-momenta of the two photons [21]:

$$
\begin{gathered}
q=\xi_{q}^{+} p_{1}+\xi_{q}^{-} p_{2}+q_{\perp} \quad p=\xi_{p}^{+} p_{1}+\xi_{p}^{-} p_{2}+p_{\perp} \\
\text { with } \\
p_{i}^{2}=0, p_{i} \cdot q_{\perp}=p_{i} \cdot p_{\perp}=0, p_{1} \cdot p_{2}=S / 2
\end{gathered}
$$


where $S$ is the square of the ee-CMS energy. The momentum fractions $\xi_{q}^{+}$, $\xi_{q}^{-}, \xi_{p}^{+}$and $\xi_{p}^{-}$and the transverse momenta can be easily calculated:

$$
\begin{array}{lll}
2 p_{1} \cdot q=S \xi_{q}^{-}=-Q^{2} & \Rightarrow & \xi_{q}^{-}=-Q^{2} / S \\
2 p_{2} \cdot q=S \xi_{q}^{+} & \Rightarrow & \xi_{q}^{+}=2 p_{2} \cdot q / S\left(\approx y_{1}\right) \\
2 p_{1} \cdot p=S \xi_{p}^{-} & \Rightarrow & \xi_{p}^{-}=2 p_{1} \cdot p / S\left(\approx y_{2}\right) \\
2 p_{2} \cdot p=S \xi_{p}^{+}=-P^{2} & \Rightarrow & \xi_{p}^{+}=-P^{2} / S \\
Q^{2}=-S \xi_{q}^{+} \xi_{q}^{-}-q_{\perp}^{2} & \Rightarrow & q_{\perp}^{2}=-Q^{2}\left(1-\xi_{q}^{+}\right) \\
P^{2}=-S \xi_{p}^{+} \xi_{p}^{-}-p_{\perp}^{2} & \Rightarrow & p_{\perp}^{2}=-P^{2}\left(1-\xi_{p}^{-}\right) \\
q_{\perp} \cdot p_{\perp} \equiv-\sqrt{q_{\perp}^{2} p_{\perp}^{2}} \cos \phi=-\sqrt{Q^{2} P^{2}\left(1-\xi_{q}^{+}\right)\left(1-\xi_{p}^{-}\right)} \cos \phi .
\end{array}
$$

Here $\phi$ is the angle between the scattering planes of the $e^{-}$and $e^{+}$in the $e^{+} e^{-}$-CMS. Obviously $\xi_{p}^{+}$is negligibly small such that we can use ${ }^{4}$

$$
p=\xi_{p}^{-} p_{2}+p_{\perp} .
$$

(On the other hand $\xi_{q}^{-}$cannot be neglected in the Bjorken limit.) In the $e^{+} e^{-}$-CMS the 4-momenta of the incoming leptons can be written as $p_{1}=(E, 0,0, E)$ and $p_{2}=(E, 0,0,-E)$ where $E=\frac{\sqrt{S}}{2}$ (neglecting terms of the order $\left.\mathcal{O}\left(\frac{m_{\mathrm{e}}^{2}}{S}\right)\right)$. Since the transverse 4 -vector is given by $p_{\perp}=\left(0, p_{\perp_{x}}, p_{\perp_{y}}, 0\right)$ we can infer from Eq. (4.72) that $E_{\gamma}=\xi_{p}^{-} E$, i.e., in the $e^{+} e^{-}$-CMS $\xi_{p}^{-}$is the energy fraction of the lepton energy transferred to the photon. For a real $\left(P^{2}=0\right)$ photon we recover the familiar relation $p=\xi_{p}^{-} p_{2}$ between the 4-momenta $p$ and $p_{2}$ of the collinearly radiated photon and its (massless) "parent" lepton, respectively.

Before turning to the photon density matrix elements in the Bjorken limit it is helpful to relate the variable $\nu \equiv p \cdot q$ to $\xi_{q}^{+}, \xi_{p}^{-}$and the transverse momenta:

$$
2 \nu=S \xi_{p}^{-} \xi_{q}^{+}(1+\rho), \rho \equiv \frac{2 p_{\perp} \cdot q_{\perp}}{S \xi_{p}^{-} \xi_{q}^{+}} \approx-2 \sqrt{x \delta\left(1-\xi_{q}^{+}\right)\left(1-\xi_{p}^{-}\right)} \cos \phi
$$

where $\rho \propto \sqrt{\delta}$ is small in the Bjorken limit. Employing Eq. (4.73) we find in addition

$$
y_{1} \equiv \frac{\nu}{p \cdot p_{1}}=\xi_{q}^{+}(1+\rho), \quad y_{2} \equiv \frac{\nu}{q \cdot p_{2}}=\xi_{p}^{-}(1+\rho) .
$$

Introducing the variables $\omega_{1} \equiv q \cdot\left(p_{1}+p_{2}\right) / \sqrt{S}=\frac{\sqrt{S}}{2}\left(\xi_{q}^{-}+\xi_{q}^{+}\right)$and $\omega_{2} \equiv p \cdot\left(p_{1}+p_{2}\right) / \sqrt{S}=\frac{\sqrt{S}}{2}\left(\xi_{p}^{-}+\xi_{p}^{+}\right)$the phase space can be written as

\footnotetext{
${ }^{4}$ Of course, in the calculation of quantities which are themselves small of the order $P^{2}$ (e.g. $p_{\perp}^{2}$ in the $e^{+} e^{-}-\mathrm{CMS}$ or $p_{2}^{2} \perp$ in the $\gamma \gamma$-CMS) $\xi_{p}^{+}$must be taken into account.
} 
[21] [Eq.(5.15b)] (up to terms of the order $\left.\mathcal{O}\left(\frac{m_{\mathrm{e}}^{2}}{S}\right)\right)$

$$
\begin{aligned}
\frac{d^{3} p_{1}^{\prime}}{E_{1}^{\prime}} \frac{d^{3} p_{2}^{\prime}}{E_{2}^{\prime}} & =\frac{2 \pi}{S} d Q^{2} d P^{2} d \omega_{1} d \omega_{2} d \phi=\frac{\pi}{2} d Q^{2} d \xi_{q}^{+} d P^{2} d \xi_{p}^{-} d \phi \\
& =\frac{\pi}{2} d Q^{2} d y_{1} d P^{2} d \xi_{p}^{-} d \phi(1+\mathcal{O}(\rho))
\end{aligned}
$$

where the third equality can be understood with the help of Eq. (4.74).

In the Bjorken limit the photon density matrix elements in Eq. (2.21) can be cast in a very compact form using the symmetric notation $Q_{1}^{2}=Q^{2}$ and $Q_{2}^{2}=P^{2}$ :

$$
\begin{aligned}
2 \rho_{\mathrm{i}}^{++} & =\frac{2}{y_{i}} Q_{i}^{2}\left[\frac{1+\left(1-y_{i}\right)^{2}}{y_{i}} \frac{1}{Q_{i}^{2}}-\frac{2 m_{\mathrm{e}}^{2} y_{i}}{Q_{i}^{4}}\right]+\mathcal{O}(\delta) \\
\rho_{\mathrm{i}}^{00} & =\frac{2}{y_{i}} Q_{i}^{2}\left[\frac{2\left(1-y_{i}\right)}{y_{i}} \frac{1}{Q_{i}^{2}}\right]+\mathcal{O}(\delta) \\
\left|\rho_{\mathrm{i}}^{+-}\right| & =\frac{1}{y_{i}} Q_{i}^{2}\left[\frac{2\left(1-y_{i}\right)}{y_{i}} \frac{1}{Q_{i}^{2}}-\frac{2 m_{\mathrm{e}}^{2} y_{i}}{Q_{i}^{4}}\right]+\mathcal{O}(\delta) .
\end{aligned}
$$

These results have to be expressed by the independent integration variables $Q^{2}, y_{1}, P^{2}$, and $\xi_{p}^{-}$. Furthermore, from here on we identify $y_{1} \equiv y$.

Obviously $\rho_{2}^{++}$and $\rho_{2}^{00}$ are proportional to the flux factors of transverse respectively longitudinal photons in Eq. (4.67)

$$
\begin{aligned}
2 \rho_{2}^{++} & =\frac{2}{\xi_{p}^{-}} P^{2} \frac{2 \pi}{\alpha} f_{\gamma_{T} / e}\left(\xi_{p}^{-}, P^{2}\right)+\mathcal{O}(\rho) \\
\rho_{2}^{00} & =\frac{2}{\xi_{p}^{-}} P^{2} \frac{2 \pi}{\alpha} f_{\gamma_{L} / e}\left(\xi_{p}^{-}, P^{2}\right)+\mathcal{O}(\rho)
\end{aligned}
$$

and similarly we can write

$$
\begin{aligned}
2 \rho_{1}^{++} & =\frac{2}{y} Q^{2}\left[\frac{1+(1-y)^{2}}{y} \frac{1}{Q^{2}}\right], \quad \rho_{1}^{00}=\frac{2}{y} Q^{2}\left[\frac{2(1-y)}{y} \frac{1}{Q^{2}}\right] \\
\varepsilon & =\frac{\rho_{1}^{00}}{2 \rho_{1}^{++}}=\frac{2(1-y)}{1+(1-y)^{2}}
\end{aligned}
$$

where we have discarded the mass terms due to $Q^{2} \gg m_{\mathrm{e}}^{2}$.

Inserting Eqs. (4.77), (4.76), and (4.75) into Eq. (4.69) one straightforwardly obtains (using $\frac{F_{\gamma \gamma}}{F_{e e}}=2 \nu \bar{\beta} / S=\xi_{p}^{-} y \bar{\beta}$ )

$$
\begin{aligned}
d \sigma=d Q^{2} d y d P^{2} d \xi_{p}^{-} \frac{d \phi}{2 \pi}\{ & \left.f_{\gamma_{T} / e}\left(\xi_{p}^{-}, P^{2}\right) \frac{d \sigma\left(e \gamma_{\mathrm{T}} \rightarrow e X\right)}{d y d Q^{2}}\right|_{\hat{S}=\xi_{p}^{-} S}+\left[\gamma_{\mathrm{T}} \rightarrow \gamma_{\mathrm{L}}\right] \\
& +\mathcal{O}(\rho)+\text { interference-terms }\}
\end{aligned}
$$


with the cross sections for deep inelastic electron-photon scattering given by [cf. Eq. (4.66)]

$$
\begin{aligned}
\left.\frac{d \sigma\left(e \gamma_{\mathrm{T}, \mathrm{L}} \rightarrow e X\right)}{d y d Q^{2}}\right|_{\hat{S}=\xi_{p}^{-} S}= & \frac{4 \pi^{2} \alpha}{Q^{2}} \frac{\alpha}{2 \pi}\left[\frac{1+(1-y)^{2}}{y} \frac{1}{Q^{2}}\right] \times \\
& {\left[2 x F_{1}^{\gamma_{\mathrm{T}, \mathrm{L}}}\left(x, Q^{2}\right)+\varepsilon F_{\mathrm{L}}^{\gamma_{\mathrm{T}, \mathrm{L}}}\left(x, Q^{2}\right)\right] }
\end{aligned}
$$

where $\varepsilon$ can be found in Eq. (4.78). Note that only two of the variables $x$, $y$, and $Q^{2}$ are independent since they are related via $Q^{2}=\hat{S} x y$. The terms proportional to $\rho$ vanish after $\phi$-integration like terms of the order $\mathcal{O}(\delta)$.

Unfortunately, the interference terms are proportional to $\cos \bar{\phi}$ and $\cos 2 \bar{\phi}$ where $\bar{\phi}$ is the angle of the electron scattering planes in the $\gamma \gamma$-CMS while $\phi$ is the angle of the electron scattering planes in the $e^{+} e^{-}$-CMS (三 laboratory system). However, we show below that

$$
\cos \bar{\phi}=\cos \phi \times(1+\mathcal{O}(\rho)) .
$$

Therefore, we can also get rid of the interference terms (proportional to $\cos \bar{\phi} \approx \cos \phi$ ) by integrating over $\phi$, leaving a remainder of the order $\mathcal{O}(\sqrt{\delta})$. The latter becomes obvious if we remember that the variable $\rho$ defined in (4.73) is proportional to $\sqrt{\delta} \cos \phi$ such that terms $\rho \cos \phi$ occurring for example in Eq. (4.81) are proportional to $\sqrt{\delta} \cos ^{2} \phi$ which do not vanish by integrating over $\phi$.

Before calculating $\cos \bar{\phi}$ let us state the final factorization formula:

$$
\begin{aligned}
\frac{d \sigma(e e \rightarrow e e X)}{d y d Q^{2} d \xi_{p}^{-} d P^{2}}= & \left.f_{\gamma_{T} / e}\left(\xi_{p}^{-}, P^{2}\right) \frac{d \sigma\left(e \gamma_{\mathrm{T}} \rightarrow e X\right)}{d y d Q^{2}}\right|_{\hat{S}=\xi_{p}^{-} S}+\mathcal{O}(\delta) \\
& +\left.f_{\gamma_{L} / e}\left(\xi_{p}^{-}, P^{2}\right) \frac{d \sigma\left(e \gamma_{\mathrm{L}} \rightarrow e X\right)}{d y d Q^{2}}\right|_{\hat{S}=\xi_{p}^{-} S}+\mathcal{O}(\delta) \\
& +\mathcal{O}(\sqrt{\delta}) .
\end{aligned}
$$

To complete our derivation we still have to show that $\cos \bar{\phi}=\cos \phi+$ $\mathcal{O}(\rho)$ where $\cos \bar{\phi}$ is given by Eq. (A.4) in [21]

$$
\cos \bar{\phi} \equiv \frac{-p_{1 \perp} \cdot p_{2}}{\sqrt{p_{1}{ }_{\perp}^{2} p_{2}^{2}}} \quad \text { with } \quad p_{i_{\perp}}^{\mu}=-p_{i_{\nu}} R^{\mu \nu}(q, p)
$$

and where $R^{\mu \nu}(q, p)$ has been defined in Eq. (2.12). Using the decomposition in Eq. (4.70) it is straightforward to obtain

$$
\begin{aligned}
p_{1 \perp} \cdot p_{2 \perp} & =\frac{p_{\perp} \cdot q_{\perp}}{\xi_{p}^{-} \xi_{q}^{+}} \\
p_{1 \perp}^{2} & =-\frac{Q^{2}}{\xi_{q}^{+2}}\left(1-\xi_{q}^{+}\right)+\mathcal{O}(\rho) \\
p_{2}^{2} & =-\frac{P^{2}}{\xi_{p}^{-2}}\left(1-\xi_{p}^{-}\right)+\mathcal{O}(\rho) .
\end{aligned}
$$


Inserting these relations into Eq. (4.83) and comparing with the definition of $\cos \phi$ in Eq. (4.71) we find the above stated result

$$
\cos \bar{\phi}=\frac{-p_{\perp} \cdot q_{\perp}}{\sqrt{p_{\perp}^{2} q_{\perp}^{2}}}+\mathcal{O}(\rho)=\cos \phi+\mathcal{O}(\rho) .
$$

A few comments are in order:

- In the limit $P^{2} \rightarrow P_{\min }^{2} \approx 0$ the contributions from longitudinal target photons have to vanish since a real photon has only two transverse physical degrees of freedom and one recovers Eq. (4.65). Indeed, the doubly virtual box results for $\sigma_{\mathrm{TL}}$ and $F_{2}^{\gamma_{\mathrm{L}}}\left(x, Q^{2}\right)$ vanish like $\propto P^{2} / m_{q}^{2}$ where $m_{q}$ is the quark mass. Clearly, the box expressions are not reliable in this limit. However, assuming that the structure functions of a longitudinal target photon vanish like $P^{2} / \Lambda^{2}$ where $\Lambda$ is a typical hadronic scale, say $\Lambda=m_{\rho}$, we obtain a non-zero result because $f_{\gamma_{L} / e} \propto 1 / P^{2}$ which is, however, negligible compared to the contribution from transverse target photons.

- The factorized result in Eq. (4.82) is valid up to terms $\mathcal{O}(\sqrt{\delta})$ which formally go to zero in the Bjorken limit. However, for practical purposes it is not clear when $\delta=x P^{2} / Q^{2}$ is small enough for Eq. (4.82) to be a good approximation of the exact cross section in Eq. (2.22). Here, a numerical comparison of the factorization formula with the exact cross section would be interesting.

- As is well known, a similar factorization into transverse and longitudinal photon fluxes also holds in $e p$ scattering such that the parton distributions for transverse and longitudinal virtual photons are useful also in the description of these reactions. Here 'transverse' and 'longitudinal' refer to the $\gamma^{\star} p$-CMS.

- One should keep in mind that the factorization discussed here only applies to two-photon processes as specified in the introduction and in Sec. 2.1.

\section{CONCLUSIONS}

In this work two-photon processes and their relation to the structure of real and virtual photons have been reviewed. Sec. 2 contains a comprehensible introduction to two-photon processes including a re-calculation of the virtual photon-photon box $\left(\gamma^{\star}\left(Q^{2}\right) \gamma^{\star}\left(P^{2}\right) \rightarrow q \bar{q}\right)$. Thereby two discrepancies in the literature could be clarified. A hopefully useful collection of several limits of the most general results has been provided in the Appendix A. Many of these results are wide-spread over the literature with different notations and conventions. 
In the 'generalized' $\left(P^{2} \neq 0\right)$ Bjorken limit, which practically means $P^{2} \ll Q^{2}$, two-photon processes can be described in terms of virtual photon structure functions. In contrast to the case of real target photons with only transverse (= spin-averaged) degrees of freedom one can distinguish between spin-averaged, transverse and longitudinal target photons. The latter two being more directly related to observable quantities. In Sec. 3 virtual photon structure functions have been defined for spin-averaged, transverse and longitudinal target photons and their relation has been discussed.

Finally, we have demonstrated the factorization of two-photon processes in the generalized Bjorken limit in Sec. 4. The factorization is into fluxes of transverse and longitudinal target photons times the corresponding cross sections for deep inelastic scattering off these targets.

These results being model independent may serve as a starting point for parton model calculations. In the parton model the partonic content of both spin-averaged and transverse photons is governed by inhomogeneous evolution equations. The difference is in the boundary conditions. On the other hand, parton distributions of longitudinal target photons obey homogeneous evolution equations. Since the transverse and longitudinal photon fluxes are different it appears to be advantageous to analyze the parton content of transverse and longitudinal target photons. This is also supported by the fact that a similar factorization holds in $e p$ scattering. Measurements of triple differential dijet cross sections at the $e p$ collider HERA indicate that the contributions from resolved longitudinal photons in addition to transverse resolved photons improve the description of the data [50]. Moreover, the $y$-dependence of these data favors a resolved $\gamma_{\mathrm{L}}$ contribution instead of simply enhancing the $\gamma_{\mathrm{T}}$ resolved processes. On the other hand, due to experimental limitations measurements of DIS $_{e \gamma}$ are performed at small $y$ where the transverse and longitudinal fluxes are

equal. However, it is not the spin-averaged combination ' $\gamma_{\mathrm{T}}-\frac{1}{2} \gamma_{\mathrm{L}}$ ' which is probed but the effective combination ' $\gamma_{\mathrm{T}}+\gamma_{\mathrm{L}}$ '.

So far models for the parton content of virtual photons $[8,15,6]$ do not clearly distinguish between 'transverse' and 'spin-averaged'. Furthermore, the only analysis of the parton content of longitudinal target photons [22] is valid in the perturbative realm $P^{2} \gg \Lambda^{2}$ where $\Lambda$ is a typical hadronic scale and does not take into account any hadronic contributions. This leaves room for future investigations of the parton content of virtual photons.

\section{APPENDIX A: LIMITS OF THE DOUBLY VIRTUAL BOX}

Due to the particular choice of variables, the results in Eqs. (2.41)-(2.48) are especially well suited for deriving various important limits wide-spread over the literature.

- Most important is the Bjorken limit $\left(Q^{2} \rightarrow \infty, \nu \rightarrow \infty, x=\right.$ const $)$ in 
which we can study structure functions of the real and virtual photon.

- Another important case is the real photon $P^{2}=0(\delta=0)$ case in which the general virtual box results in $(2.41)-(2.48)$ reduce to the standard box-diagram $\gamma^{\star}\left(Q^{2}\right) \gamma \rightarrow q \bar{q}$ expressions for a real photon $\gamma \equiv \gamma\left(P^{2}=0\right)$. Keeping the full mass dependence in (2.41)-(2.48) we obtain expressions relevant for the heavy quark contribution to the photon structure functions.

- Finally, the general light quark mass limit (for arbitrary $P^{2}, Q^{2}$ ) can be easily obtained from (2.41)-(2.48) by setting $m=0(\lambda=0, \beta=1)$ and needs no separate discussion.

In the following, our main concern will lie on the Bjorken limit. Practically this limit means that $Q^{2}$ is much larger than the other scales $m^{2}$ and $P^{2}$. Beside the general case $m^{2}, P^{2} \ll Q^{2}$ which will be studied in Section A.1 additional orderings $m^{2}=0, P^{2} \ll Q^{2}$ (Section A.2) and $P^{2}=0, m^{2} \ll Q^{2}$ (Section A.3) are of interest and the expressions further simplify under these circumstances. One must be careful in handling these limits because for some of the box cross sections the result depends on which of the two limits $m \rightarrow 0$ and $P^{2} \rightarrow 0$ is taken first. For example, below we will find the following asymptotic expressions for $\sigma_{\mathrm{TL}}$ derived from (2.41)-(2.48):

$$
0=\lim _{m^{2} \rightarrow 0} \lim _{P^{2} \rightarrow 0} \sigma_{\mathrm{TL}} \neq \lim _{P^{2} \rightarrow 0} \lim _{m^{2} \rightarrow 0} \sigma_{\mathrm{TL}}=N_{c} e_{q}^{4} \frac{4 \pi \alpha^{2}}{Q^{2}} 4 x^{2}(1-x)
$$

Mathematically, the origin of such a behavior is easily identified. Terms like $\frac{4 x \delta}{4 x \delta+\lambda \bar{\beta}^{2}}$ occurring in (2.41)-(2.48) (not only as the argument of the logarithm) require a careful treatment. In general they are not negligible even for small $m^{2}$ and $P^{2}$ and, viewed as a function of $m^{2}$ and $P^{2}$, they are discontinuous at $\left(m^{2}, P^{2}\right)=(0,0)$. Finally, in Section A.3 we also derive expressions for the heavy quark contributions to the photon structure functions in the real photon limit by setting $P^{2}=0(\delta=0)$ but keeping the full mass dependence in (2.41)-(2.48).

All results for the photon-photon cross sections will be given for a single quark with charge $e_{q}$ and mass $m$. The photon structure functions $F_{\mathrm{i}}$, (i $=$ $1,2, \mathrm{~L})$ can be obtained from these expressions with help of the relations in Section 3 which simplify in the Bjorken limit $\left(P^{2} \ll Q^{2} \Rightarrow \bar{\beta} \simeq 1\right)$ :

$$
\begin{aligned}
F_{2}^{\gamma_{\mathrm{T}}} & =\frac{Q^{2}}{4 \pi^{2} \alpha} \sigma_{2 \mathrm{~T}}, & F_{\mathrm{L}}^{\gamma_{\mathrm{T}}} & =\frac{Q^{2}}{4 \pi^{2} \alpha} \sigma_{\mathrm{LT}} \\
F_{2}^{\gamma_{\mathrm{L}}} & =\frac{Q^{2}}{4 \pi^{2} \alpha} \sigma_{2 \mathrm{~L}}, & F_{\mathrm{L}}^{\gamma_{\mathrm{L}}} & =\frac{Q^{2}}{4 \pi^{2} \alpha} \sigma_{\mathrm{LL}}
\end{aligned}
$$

with $\sigma_{2 \mathrm{~T}}=\sigma_{\mathrm{TT}}+\sigma_{\mathrm{LT}}, \sigma_{2 \mathrm{~L}}=\sigma_{\mathrm{TL}}+\sigma_{\mathrm{LL}}$. The commonly utilized expressions for a spin-averaged target photon are given by

$$
F_{\mathrm{i}}^{<\gamma>}=F_{\mathrm{i}}^{\gamma_{\mathrm{T}}}-\frac{1}{2} F_{\mathrm{i}}^{\gamma_{\mathrm{L}}},(\mathrm{i}=1,2, \mathrm{~L}) \text {. }
$$


Finally, the structure function $F_{1}$ can be deduced from $F_{\mathrm{L}}=F_{2}-2 x F_{1}$. Since the structure functions are (apart from the normalization factor $Q^{2} / 4 \pi^{2} \alpha$ ) simple linear combinations of the photon-photon cross sections they will only be written out in some special cases.

\section{A.1. General Bjorken Limit: $m^{2}, P^{2} \ll Q^{2}$}

In the general Bjorken limit the normalization factor $N$ given in Eq. (2.40) and the logarithm $L$ from Eq. (2.39) are given by

$$
\begin{gathered}
N=4 \pi N_{c} e_{q}^{4} \frac{4 \pi \alpha^{2}}{Q^{2}} x(1+\mathcal{O}(\delta)) \\
L=\ln \frac{4}{4 x \delta+\lambda}+\mathcal{O}(\delta, \lambda) .
\end{gathered}
$$

Keeping this in mind and using $\beta=1+\mathcal{O}(\lambda), \bar{\beta}=1+\mathcal{O}(\delta)$ the following results can be easily deduced from Eqs. (2.41)-(2.48):

$$
\begin{aligned}
\sigma_{\mathrm{TT}} & =\tilde{N}_{q}\left\{\left[x^{2}+(1-x)^{2}\right] L+4 x(1-x)-1-\frac{4 x \delta}{4 x \delta+\lambda}+\mathcal{O}(\delta, \lambda)\right\} \\
\sigma_{\mathrm{LT}} & =\tilde{N}_{q}\{4 x(1-x)+\mathcal{O}(\delta, \lambda)\} \\
\sigma_{\mathrm{TL}} & =\tilde{N}_{q}\left\{4 x(1-x) \frac{4 x \delta}{4 x \delta+\lambda}+\mathcal{O}(\delta, \lambda)\right\} \\
\tau_{\mathrm{TT}} & =\tilde{N}_{q}\left\{-2 x^{2}+\mathcal{O}(\delta, \lambda)\right\} \\
\tau_{\mathrm{TT}}^{\mathrm{a}} & =\tilde{N}_{q}\left\{(2 x-1) L+3-4 x-\frac{4 x \delta}{4 x \delta+\lambda}+\mathcal{O}(\delta, \lambda)\right\}
\end{aligned}
$$

with $\tilde{N}_{q}=N_{c} e_{q}^{4} \frac{4 \pi \alpha^{2}}{Q^{2}} x$ where $N_{c}=3$ is the number of colors and $e_{q}$ is the quark charge. The remaining expressions are suppressed by powers of $\delta=x P^{2} / Q^{2}$ (see Eqs. (2.41)-(2.48)).

At the price of a slightly worse approximation (at larger $x$ ) one can further use

$$
\begin{gathered}
\frac{4 x \delta}{4 x \delta+\lambda}=\frac{P^{2} x(1-x)}{P^{2} x(1-x)+m^{2}}+\mathcal{O}\left(\frac{P^{2}}{W^{2}}, \lambda\right), \\
L=\ln \frac{Q^{2}(1-x)}{x\left[P^{2} x(1-x)+m^{2}\right]}+\mathcal{O}\left(\frac{P^{2}}{W^{2}}, \lambda\right) .
\end{gathered}
$$

For example we can write:

$$
\begin{aligned}
\sigma_{\mathrm{TT}}=\tilde{N}_{q}\{ & {[1-2 x(1-x)] \ln \frac{Q^{2}(1-x)}{x\left[P^{2} x(1-x)+m^{2}\right]}+4 x(1-x) } \\
& \left.-1-\frac{P^{2} x(1-x)}{P^{2} x(1-x)+m^{2}}+\mathcal{O}\left(\frac{P^{2}}{W^{2}}, \lambda\right)\right\} .
\end{aligned}
$$




\section{A.2. $m^{2}=0, P^{2} \ll Q^{2}$}

In this section we consider the asymptotic virtual $\left(P^{2} \neq 0\right)$ box expressions for light quarks in the Bjorken limit for which the expressions in Eq. (A.89) further reduce. Noticing that the logarithm $L$ is given by $L=\ln \frac{Q^{2}}{P^{2} x^{2}}$ we can write in this case (neglecting terms of the order $\mathcal{O}(\delta)$ ):

$$
\begin{aligned}
& \sigma_{\mathrm{TT}} \simeq \tilde{N}_{q}\left\{\left[x^{2}+(1-x)^{2}\right] \ln \frac{Q^{2}}{P^{2} x^{2}}+4 x(1-x)-2\right\} \\
& \sigma_{\mathrm{TL}} \simeq \sigma_{\mathrm{LT}} \simeq \tilde{N}_{q}[4 x(1-x)] \\
& \tau_{\mathrm{TT}} \simeq \tilde{N}_{q}\left[-2 x^{2}\right] \\
& \tau_{\mathrm{TT}}^{\mathrm{a}} \simeq \tilde{N}_{q}\left\{(2 x-1) \ln \frac{Q^{2}}{P^{2} x^{2}}+2-4 x\right\} .
\end{aligned}
$$

(Recall the normalization factor $\tilde{N}_{q}=N_{c} e_{q}^{4} \frac{4 \pi \alpha^{2}}{Q^{2}} x$.)

Summing over $q=u, d, s$ and utilizing Eqs. (A.86) and (A.87) we recover the well known asymptotic results for the virtual $\left(P^{2} \neq 0\right)$ box structure functions for the light $q=u, d, s$ quarks in the Bjorken limit $P^{2} / Q^{2} \ll 1$ :

$$
\begin{aligned}
F_{2, \text { box }}^{\gamma_{\mathrm{T}}, \ell}\left(x, Q^{2}, P^{2}\right) & \simeq N_{c} \sum e_{q}^{4} \frac{\alpha}{\pi} x\left\{\left[x^{2}+(1-x)^{2}\right] L+8 x(1-x)-2\right\} \\
F_{2, \text { box }}^{\gamma_{\mathrm{L}}, \ell}\left(x, Q^{2}, P^{2}\right) & \simeq N_{c} \sum e_{q}^{4} \frac{\alpha}{\pi} x\{4 x(1-x)\} \\
F_{2, \text { box }}^{<\gamma>, \ell}\left(x, Q^{2}, P^{2}\right) & \simeq N_{c} \sum e_{q}^{4} \frac{\alpha}{\pi} x\left\{\left[x^{2}+(1-x)^{2}\right] L+6 x(1-x)-2\right\} .
\end{aligned}
$$

\section{A.3. Real Photon Limit: $P^{2}=0$}

For $P^{2}=0$ the virtual box results in $(2.41)-(2.48)$ reduce to the standard box-diagram $\gamma^{\star}\left(Q^{2}\right) \gamma \rightarrow q \bar{q}$ expressions for a real photon $\gamma \equiv \gamma\left(P^{2}=\right.$ $0)$.

\section{A.3.1. Heavy Quark Contribution}

The heavy quark contribution becomes, utilizing $\delta=0, \bar{\beta}=1$ and $\lambda=\frac{4 m_{h}^{2} x}{Q^{2}(1-x)}$ and the normalization factor $\tilde{N}_{h}=N_{c} e_{h}^{4} \frac{4 \pi \alpha^{2}}{Q^{2}} x$ :

$$
\begin{aligned}
\sigma_{\mathrm{TT}}= & \tilde{N}_{h} \Theta\left(\beta^{2}\right)\left\{\left[x^{2}+(1-x)^{2}+x(1-x) \frac{4 m_{h}^{2}}{Q^{2}}-x^{2} \frac{8 m_{h}^{4}}{Q^{4}}\right] \times\right. \\
& \left.\ln \frac{1+\beta}{1-\beta}+\beta\left[4 x(1-x)-1-x(1-x) \frac{4 m_{h}^{2}}{Q^{2}}\right]\right\}
\end{aligned}
$$




$$
\begin{aligned}
\sigma_{\mathrm{LT}}= & \tilde{N}_{h} \Theta\left(\beta^{2}\right)\left\{-x^{2} \frac{8 m_{h}^{2}}{Q^{2}} \ln \frac{1+\beta}{1-\beta}+\beta 4 x(1-x)\right\} \\
\tau_{\text {TT }}= & \tilde{N}_{h} \Theta\left(\beta^{2}\right)\left\{\left[-x^{2} \frac{8 m_{h}^{2}}{Q^{2}}-x^{2} \frac{8 m_{h}^{4}}{Q^{4}}\right] \ln \frac{1+\beta}{1-\beta}\right. \\
& \left.-\beta\left[2 x^{2}+x(1-x) \frac{4 m_{h}^{2}}{Q^{2}}\right]\right\} \\
\tau_{\text {TT }}^{\mathrm{a}}= & \tilde{N}_{h} \Theta\left(\beta^{2}\right)\left\{(2 x-1) \ln \frac{1+\beta}{1-\beta}+\beta(3-4 x)\right\} .
\end{aligned}
$$

i.e., according to (A.87) (or (A.86))

$$
\begin{aligned}
F_{2, \text { box }}^{\gamma, h}\left(x, Q^{2}\right) & =3 e_{h}^{4} \frac{\alpha}{\pi} x \Theta\left(\beta^{2}\right)\left\{\left[x^{2}+(1-x)^{2}+x(1-3 x) \frac{4 m_{h}^{2}}{Q^{2}}\right.\right. \\
& \left.\left.-x^{2} \frac{8 m_{h}^{4}}{Q^{4}}\right] \ln \frac{1+\beta}{1-\beta}+\beta\left[8 x(1-x)-1-x(1-x) \frac{4 m_{h}^{2}}{Q^{2}}\right]\right\} \\
F_{\mathrm{L}, \text { box }}^{\gamma, h}\left(x, Q^{2}\right) & =3 e_{h}^{4} \frac{\alpha}{\pi} x \Theta\left(\beta^{2}\right)\left\{-x^{2} \frac{8 m_{h}^{2}}{Q^{2}} \ln \frac{1+\beta}{1-\beta}+\beta 4 x(1-x)\right\}
\end{aligned}
$$

which are the familiar massive Bethe-Heitler expressions [51] relevant for the heavy quark contributions to the structure functions of real photons (cf. [8], for example).

\section{A.3.2. Light Quark Contribution}

In the light quark sector where $\lambda \ll 1$, i.e. $m^{2} \equiv m_{q}^{2} \ll Q^{2}$, the logarithm can be written as

$$
L=\ln \frac{1+\beta}{1-\beta}=\ln \frac{Q^{2}(1-x)}{m_{q}^{2} x}+\mathcal{O}(\lambda) .
$$

and we obtain from (A.89) or (A.94) the following results (neglecting terms of the order $\mathcal{O}(\lambda))$ :

$$
\begin{aligned}
\sigma_{\mathrm{TT}} & \simeq \tilde{N}_{q}\left\{\left[x^{2}+(1-x)^{2}\right] \ln \frac{Q^{2}(1-x)}{m_{q}^{2} x}+4 x(1-x)-1\right\} \\
\sigma_{\mathrm{LT}} & \simeq \tilde{N}_{q}[4 x(1-x)] \\
\tau_{\mathrm{TT}} & \simeq \tilde{N}_{q}\left[-2 x^{2}\right] \\
\tau_{\mathrm{TT}}^{\mathrm{a}} & \simeq \tilde{N}_{q}\left\{(2 x-1) \ln \frac{Q^{2}(1-x)}{m_{q}^{2} x}+3-4 x\right\},
\end{aligned}
$$

i.e., according to (A.87) (or (A.86))

$F_{2, \text { box }}^{\gamma, \ell}\left(x, Q^{2}\right) \simeq N_{c} \sum e_{q}^{4} \frac{\alpha}{\pi} x\left\{\left[x^{2}+(1-x)^{2}\right] \ln \frac{Q^{2}(1-x)}{m_{q}^{2} x}+8 x(1-x)-1\right\}$. 
Note also that $\sigma_{\mathrm{TL}}$ vanishes like $\sigma_{\mathrm{TL}} \propto P^{2} / m_{q}^{2}$.

\section{ACKNOWLEDGMENTS}

I am grateful to E. Reya for suggesting this work and for continuous encouragement. I also wish to thank C. Sieg for a pleasant collaboration on parts of Secs. 3 and 4. This work has been supported in part by the 'Bundesministerium für Bildung und Forschung', Berlin/Bonn.

\section{REFERENCES}

[1] S. J. Brodsky, T. Kinoshita, and H. Terazawa, Phys. Rev. Lett. 27, 280 (1971).

T. F. Walsh, Phys. Lett. B36, 121 (1971).

[2] R. Nisius, Phys. Rept. 332, 165 (2000).

[3] M. Krawczyk, A. Zembrzuski, and M. Staszel, Phys. Rept. 345, 265 (2001).

[4] M. Glück, E. Reya, and A. Vogt, Phys. Rev. D45, 3986 (1992).

M. Glück, E. Reya, and A. Vogt, Phys. Rev. D46, 1973 (1992).

[5] P. Aurenche, M. Fontannaz, and J.-P. Guillet, Z. Phys. C64, 621 (1994).

[6] G. A. Schuler and T. Sjöstrand, Z. Phys. C68, 607 (1995).

G. A. Schuler and T. Sjöstrand, Phys. Lett. B376, 193 (1996).

[7] L. E. Gordon and J. K. Storrow, Nucl. Phys. B489, 405 (1997).

[8] M. Glück, E. Reya, and I. Schienbein, Phys. Rev. D60, 054019 (1999), Erratum: D62 (2000) 019902.

[9] M. Glück and W. Vogelsang, Z. Phys. C55, 353 (1992).

M. Glück and W. Vogelsang, Z. Phys. C57, 309 (1993).

M. Glück, M. Stratmann, and W. Vogelsang, Phys. Lett. B337, 373 (1994).

[10] M. Stratmann and W. Vogelsang, Phys. Lett. B386, 370 (1996).

[11] M. Glück, E. Reya, and C. Sieg, Phys. Lett. B503, 285 (2001).

M. Glück, E. Reya, and C. Sieg, Eur. Phys. J. C20, 271 (2001). 
[12] M. Stratmann and W. Vogelsang, Nucl. Phys. Proc. Suppl. 82, 400 (2000).

M. Stratmann and W. Vogelsang, Z. Phys. C74, 641 (1997).

M. Stratmann, Determining the spin structure of the photon at future colliders, talk presented at the 8th International Workshop on Deep Inelastic Scattering and QCD (DIS 2000), Liverpool, England, 25-30 Apr 2000, hep-ph/0006285.

[13] F. M. Borzumati and G. A. Schuler, Z. Phys. C58, 139 (1993).

[14] M. Drees and R. M. Godbole, Phys. Rev. D50, 3124 (1994).

[15] M. Glück, E. Reya, and M. Stratmann, Phys. Rev. D51, 3220 (1995).

[16] T. Uematsu and T. F. Walsh, Phys. Lett. B101, 263 (1981). T. Uematsu and T. F. Walsh, Nucl. Phys. B199, 93 (1982).

[17] G. Rossi, Phys. Rev. D29, 852 (1984).

[18] G. Rossi, Virtual Photon Structure Functions in Quantum Chromodynamics, Ph.D. thesis, UC San Diego (1983), UC San Diego report UCSD-10P10-227 (unpublished).

[19] W. Ibes and T. F. Walsh, Phys. Lett. B251, 450 (1990).

[20] K. Sasaki and T. Uematsu, Phys. Rev. D59, 114011 (1999). K. Sasaki and T. Uematsu, Phys. Lett. B473, 309 (2000). K. Sasaki and T. Uematsu, Eur. Phys. J. C20, 283 (2001).

[21] V. M. Budnev, I. F. Ginzburg, G. V. Meledin, and V. G. Serbo, Phys. Rep. 15, 181 (1975).

[22] J. Chýla, Phys. Lett. B488, 289 (2000).

J. Chýla and M. Taševský, Eur. Phys. J. C16, 471 (2000).

J. Chýla and M. Taševský, Eur. Phys. J. C18, 723 (2001).

[23] C. Friberg and T. Sjöstrand, Phys. Lett. B492, 123 (2000).

[24] R. Bhattacharya, J. Smith, and G. Grammer, Phys. Rev. D15, 3267 (1977).

[25] R. W. Brown and I. J. Muzinich, Phys. Rev. D4, 1496 (1971).

[26] C. E. Carlson and W.-K. Tung, Phys. Rev. D4, 2873 (1971).

[27] A. V. Manohar, An introduction to spin dependent deep inelastic scattering, lectures given at Lake Louise Winter Inst., Lake Louise, Canada, Feb 23-29, 1992, hep-ph/9204208. 
[28] K. Sasaki, J. Soffer, and T. Uematsu, Phys. Lett. B522, 22 (2001).

[29] C. Berger and W. Wagner, Phys. Rep. 146, 1 (1987).

[30] S. Wolfram, Mathematica - Ver. 3 or higher, Wolfram Research (1997).

[31] M. Jamin and M. E. Lautenbacher, Comput. Phys. Commun. 74, 265 (1993).

[32] S. Kretzer and I. Schienbein, Phys. Rev. D56, 1804 (1997).

[33] I. Schienbein, Phys. Rev. D59, 013001 (1999).

[34] R. Nisius and M. H. Seymour, Phys. Lett. B452, 409 (1999).

[35] M. Glück, E. Reya, and I. Schienbein, Phys. Rev. D63, 074008 (2001).

[36] I. Schienbein, Heavy quark production in CC and NCDIS and the structure of real and virtual photons in NLO QCD, Ph.D. thesis, University of Dortmund (2001), hep-ph/0110292.

[37] S. J. Brodsky, F. Hautmann, and D. E. Soper, Phys. Rev. D56, 6957 (1997).

S. J. Brodsky, F. Hautmann, and D. E. Soper, Phys. Rev. Lett. 78, 803 (1997), Erratum: 79 (1997) 3544.

[38] J. Bartels, A. De Roeck, and H. Lotter, Phys. Lett. B389, 742 (1996).

J. Bartels, A. De Roeck, C. Ewerz, and H. Lotter, hep-ph/9710500.

J. Bartels, C. Ewerz, and R. Staritzbichler, Phys. Lett. B492, 56 (2000).

[39] J. Kwieciński and L. Motyka, Phys. Lett. B462, 203 (1999).

J. Kwieciński and L. Motyka, Eur. Phys. J. C18, 343 (2000).

[40] V. S. Fadin, E. A. Kuraev, and L. N. Lipatov, Phys. Lett. B60, 50 (1975).

E. A. Kuraev, L. N. Lipatov, and V. S. Fadin, Sov. Phys. JETP 44, 443 (1976).

E. A. Kuraev, L. N. Lipatov, and V. S. Fadin, Sov. Phys. JETP 45, 199 (1977).

[41] I. I. Balitsky and L. N. Lipatov, Sov. J. Nucl. Phys. 28, 822 (1978).

[42] P. Achard et al., L3 Collaboration, Phys. Lett. B531, 39 (2002).

[43] G. Abbiendi et al., OPAL Collaboration, Measurement of the hadronic cross-section for the scattering of two virtual photons at LEP, hepex/0110006. 
[44] M. Cacciari, V. Del Duca, S. Frixione, and Z. Trócsányi, JHEP 02, 029 (2001).

[45] S. J. Brodsky, V. S. Fadin, V. T. Kim, L. N. Lipatov, and G. B. Pivovarov, High-energy asymptotics of photon photon collisions in $Q C D$ (2001), talk given at the 14th International Workshop on PhotonPhoton Collisions (Photon 2001), Ascona, Switzerland, 2-7 Sep 2001, hep-ph/0111390.

[46] T. F. Walsh and P. Zerwas, Phys. Lett. B44, 195 (1973).

P. M. Zerwas, Phys. Rev. D10, 1485 (1974).

R. L. Kingsley, Nucl. Phys. B60, 45 (1973).

V. L. Chernyak and V. G. Serbo, Nucl. Phys. B71, 395 (1974).

R. P. Worden, Phys. Lett. B51, 57 (1974).

M. A. Ahmed and G. G. Ross, Phys. Lett. B59, 369 (1975).

[47] J. C. Collins, D. E. Soper, and G. Sterman, in Perturbative Quantum Chromodynamics, edited by A. H. Mueller (World Scientific, 1989).

J. C. Collins and D. E. Soper, Ann. Rev. Nucl. Part. Sci. 37, 383 (1987).

[48] P. Hoodbhoy, R. L. Jaffe, and A. Manohar, Nucl. Phys. B312, 571 (1989).

[49] P. Mathews and V. Ravindran, Int. J. Mod. Phys. A11, 2783 (1996).

[50] K. Sedlák, H1 Collaboration, Structure of virtual photons at HERA (2001), talk given at the 14th International Workshop on PhotonPhoton Collisions (Photon 2001), Ascona, Switzerland, 2-7 Sep 2001, hep-ex/0111019.

[51] E. Witten, Nucl. Phys. B104, 445 (1976).

M. Glück and E. Reya, Phys. Lett. 83B, 98 (1979). 